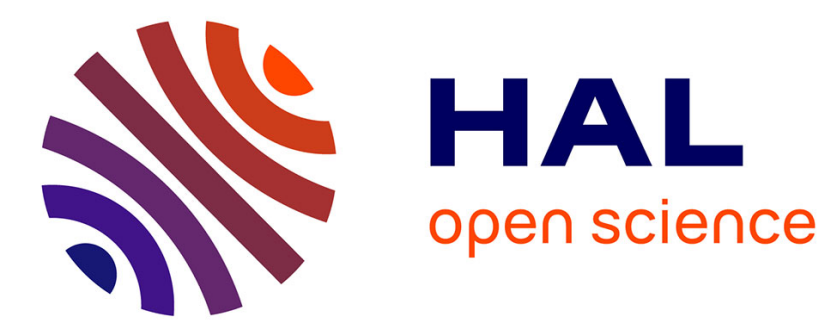

\title{
A fictitious domain method for frictionless contact problems in elasticity using Nitsche's method
}

\author{
Mathieu Fabre, Jérôme Pousin, Yves Renard
}

\section{To cite this version:}

Mathieu Fabre, Jérôme Pousin, Yves Renard. A fictitious domain method for frictionless contact problems in elasticity using Nitsche's method. SMAI Journal of Computational Mathematics, 2016, 2, pp.19-50. 10.5802/smai-jcm.8 . hal-00960996v3

\section{HAL Id: hal-00960996 https://hal.science/hal-00960996v3}

Submitted on 12 Feb 2018

HAL is a multi-disciplinary open access archive for the deposit and dissemination of scientific research documents, whether they are published or not. The documents may come from teaching and research institutions in France or abroad, or from public or private research centers.
L'archive ouverte pluridisciplinaire HAL, est destinée au dépôt et à la diffusion de documents scientifiques de niveau recherche, publiés ou non, émanant des établissements d'enseignement et de recherche français ou étrangers, des laboratoires publics ou privés. 


\title{
A fictitious domain method for frictionless contact problems in elasticity using Nitsche's method
}

\author{
Mathieu Fabre *, Jérôme Pousin ${ }^{\dagger}$, Yves Renard ${ }^{\ddagger}$
}

\begin{abstract}
In this paper, we develop and analyze a finite element fictitious domain approach based on Nitsche's method for the approximation of frictionless contact problems of two deformable elastic bodies. In the proposed method, the geometry of the bodies and the boundary conditions, including the contact condition between the two bodies, are described independently of the mesh of the fictitious domain. We prove that the optimal convergence is preserved. $\mathrm{Nu}-$ merical experiments are provided which confirm the correct behavior of the proposed method.
\end{abstract}

\section{Introduction}

In the vast majority of finite element software, the contact conditions between deformable solids are taken into account through the introduction of Lagrange multipliers and/or penalization terms. The multipliers, which generally approximate the contact stresses, represent some additional unknowns. The approximated problem is then solved in a coupled way or iteratively on the multiplier using Uzawa's algorithm (see e.g. [27]). Recently in [5, 6], it has been proposed an extension to the contact conditions of Nitsche's method $[24,11,17]$ which was originally dedicated to Dirichlet's condition. This method combines the advantages of both the penalty and Lagrange multiplier methods since it remains consistent, optimal and avoid the use of multipliers.

In a fictitious domain framework, this paper aims to adapt Nitsche's method to the case of frictionless contact of two elastic solids with the small deformations hypothesis. Frictionless contact is considered to keep the presentation as simpler as possible. However, the analysis extends without additional difficulties to the case of Tresca friction, in a similar way as in [7]. One of the advantages of the fictitious domain approach comes from the possibility to work with structured meshes regardless of the complexity of the geometry of the bodies and of the potential contact zone. This approach is particularly advantageous in the case of free boundary problems such as shape optimization and fluid-structure interaction. In that case, it prevents the consecutive remeshing which can be very costly, in particular for three-dimensional problems, and which may also generates some instabilities. More generally, a fictitious domain method may be used in the presence of complex or moving geometries to avoid meshing them.

The fictitious domain approach we consider in this work is the one using "cut elements" which is currently a subject of growing interest and is closely related to XFem approach introduced in [21] and widely studied since then (see for instance $[20,16,26,4,23]$ ). The case of a body with a

\footnotetext{
${ }^{*}$ Univ Lyon, INSA Lyon, CNRS UMR 5208, ICJ, F-69621, Villeurbanne, France. email: Mathieu.Fabre@insa-lyon.fr

${ }^{\dagger}$ Univ Lyon, INSA Lyon, CNRS UMR 5208, ICJ, F-69621, Villeurbanne, France. email: Jerome.Pousin@insa-lyon.fr

${ }^{\ddagger}$ Univ Lyon, INSA Lyon, CNRS UMR 5208, ICJ, UMR 5259, LaMCoS, F-69621, Villeurbanne, France. email: Yves.Renard@insa-lyon.fr
} 
Dirichlet (or transmission) condition with the use of cut-elements is studied in [16] when Lagrange multipliers and a Barbosa-Hughes stabilization are used, and in $[14,4,1]$ when Nitsche's method and an additional interior penalty stabilization are considered. This fictitious domain method is to be compared with more classical strategies (see $[19,13,12,25,2]$ and the references therein) where the elements are not cut. These more classical strategies offer the possibility to leave unchanged the stiffness matrix of the problem. The boundary conditions are then prescribed via additional penalty and Lagrange multiplier terms. However, in classical strategies, it is often quite difficult to obtain an optimal method regarding the convergence order which easily takes into account both Dirichlet and Neumann conditions. The Fictitious domain method with cut elements allows to consider both Dirichlet and Neumann conditions in a rather standard way. The main price to pay is the adaptation of integration methods on cut elements.

In that context of cut elements, our study is focused on the case of two bodies with Nitsche's method for both the Dirichlet condition and the frictionless contact condition.

The outline of the paper is the following. In Section 2, we introduce the contact problem and the fictitious domain situation. Then, in Section 3, the finite element approximation with the use of Nitsche's method is built. In particular, a specific, parameter free stabilization technique is introduced which is necessary to guarantee the optimal rate of convergence. The properties of the approximated problem are described in Section 4 including the existence and uniqueness of a solution to the discrete problem, the consistency and the a priori error analysis. Finally, in Section 5, some two and three-dimensional Hertz-type numerical experiments are presented which illustrate the optimality regarding the convergence of the method.

\section{The unilateral contact problem in a fictitious domain frame- work}

An example of fictitious domain situation is illustrated in Figure 1 . Let $\Omega_{i}, 1 \leqslant i \leqslant 2$, be two possibly overlapping domains with piecewise $\mathscr{C}^{1}$ boundaries included in $\mathbb{R}^{d}, d=2$ or 3 , representing the reference configurations of two elastics bodies. Let $\Omega$ be a simple shaped polygonal fictitious domain (typically allowing the use of a structured mesh) containing both $\Omega_{1}$ and $\Omega_{2}$. The boundary $\Gamma_{1}$ of $\Omega_{1}$ (respectively $\Gamma_{2}$ of $\Omega_{2}$ ) is divided into three non overlapping parts: $\Gamma_{1, C}$ the slave potential zone of contact with meas $\left(\Gamma_{1, C}\right)>0$ (respectively $\Gamma_{2, C}$ with meas $\left(\Gamma_{2, C}\right)>0$ ); $\Gamma_{1, N}$ the Neumann part (respectively $\Gamma_{2, N}$ ) and $\Gamma_{1, D}$ the Dirichlet part with meas $\left(\Gamma_{1, D}\right)>0$ (respectively $\Gamma_{2, D}$ with meas $\left(\Gamma_{2, D}\right)>0$ ).

The two elastic bodies are subjected to volume forces $f=\left(f_{1}, f_{2}\right)$ on $\Omega_{1} \times \Omega_{2}$, to surface loads $\ell=\left(\ell_{1}, \ell_{2}\right)$ on $\Gamma_{1, N} \times \Gamma_{2, N}$ and satisfy non homogeneous boundary Dirichlet conditions on $\Gamma_{1, D} \times \Gamma_{2, D}$, the displacement being prescribed to the given value $u_{D}=\left(u_{1, D}, u_{2, D}\right)$. We assume small elastic deformation for the two bodies. The linearized strain tensor field is given by $\varepsilon(v)=\frac{1}{2}\left(\nabla v+\nabla v^{T}\right)$ and the stress tensor field $\sigma=\left(\sigma_{i j}\right)_{1 \leqslant i, j \leqslant 2}$ is given by $\sigma(v)=A \varepsilon(v)$ where $A$ is the fourth order symmetric elasticity tensor satisfying the usual uniform ellipticity and boundedness properties. Consequently, the displacement $\left(u_{1}, u_{2}\right)$ on $\Omega_{1} \times \Omega_{2}$ has to satisfy the following set of equations, apart for the contact condition which will be described later:

$$
\left\{\begin{array}{cc}
\text { Find } u=\left(u_{1}, u_{2}\right) \text { satisfying } & \\
-\operatorname{div} \sigma\left(u_{i}\right)=f_{i} & \text { in } \Omega_{i}, \\
\sigma\left(u_{i}\right)=A \varepsilon\left(u_{i}\right) & \text { in } \Omega_{i}, \\
u_{i}=u_{i, D} & \text { on } \Gamma_{i, D}, \\
\sigma\left(u_{i}\right) n_{i}=\ell_{i} & \text { on } \Gamma_{i, N} .
\end{array}\right.
$$




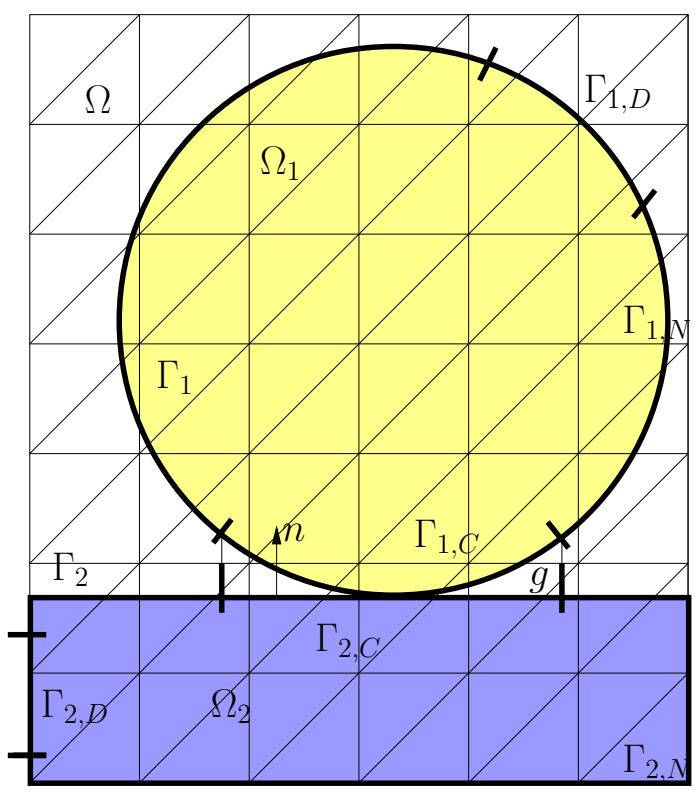

Figure 1: Example of fictitious domain situation for a contact problem between two elastics bodies with an example of structured mesh.

Now, concerning the contact conditions, let us define $\Pi$ the orthogonal projection from the slave boundary $\Gamma_{1, C}$ on the master boundary $\Gamma_{2, C}$ :

$$
\Pi: \begin{array}{ll}
\Gamma_{1, C} & \rightarrow \Gamma_{2, C} \\
x & \mapsto \Pi(x)
\end{array}
$$

In order to simplify the mathematical analysis, the operator $\Pi$ is assumed to be a $\mathscr{C}^{1}$ one to one correspondence on $\Pi\left(\Gamma_{1, C}\right)$ (this hypothesis is satisfied, for instance, when $\Gamma_{i, C}$ are convex and $\mathscr{C}^{1}$ for $\left.i \in\{1,2\}\right)$. The outward unit normal vector $n$ for the contact condition is chosen to be the one of $\Gamma_{2, C}$ :

$$
n: \begin{array}{llc}
\Gamma_{1, C} & \rightarrow & \mathbb{R}^{d} \\
x & \mapsto & n_{2}(\Pi(x)) .
\end{array}
$$

The initial gap $g$ between $\Gamma_{1, C}$ and $\Gamma_{2, C}$ is defined to be the following distance function:

$$
g: \begin{array}{ll}
\Gamma_{1, C} & \rightarrow \mathbb{R} \\
x & \mapsto(x-\Pi(x)) \cdot n .
\end{array}
$$

For $\left(v_{1}, v_{2}\right)$ a displacement field defined on $\Omega_{1} \times \Omega_{2}$, the normal jump is defined on the slave boundary $\Gamma_{1}$ for the normal displacement as follows:

$$
\llbracket v \cdot n \rrbracket=\left(v_{2} \circ \Pi-v_{1}\right) \cdot n .
$$

Concerning the normal stress, we define

$$
\sigma\left(v_{1}\right) n_{1}=-\sigma_{n}\left(v_{1}\right) n+\sigma_{t}\left(v_{1}\right) \quad \text { with } \sigma_{n}\left(v_{1}\right)=-\sigma\left(v_{1}\right) n_{1} \cdot n
$$

and

$$
\sigma\left(v_{2} \circ \Pi\right) n_{2} \circ \Pi=\sigma_{n}\left(v_{2} \circ \Pi\right) n+\sigma_{t}\left(v_{2} \circ \Pi\right) \quad \text { with } \sigma_{n}\left(v_{2} \circ \Pi\right)=\sigma\left(v_{2} \circ \Pi\right) n_{2} \circ \Pi \cdot n .
$$


This allows to define the normal stress jump as

$$
\llbracket \sigma(u) n \rrbracket=\sigma\left(u_{1}\right) n_{1}+\sigma\left(u_{2} \circ \Pi\right) n_{2} \circ \Pi\left|\operatorname{det}\left(J_{\Pi}\right)\right|,
$$

with $J_{\Pi}$ the Jacobian matrix of $\Pi$. This latter expression is derived accordingly with Newton's second law (action-reaction principle) which is expressed on arbitrary elementary surfaces (see Figure 2):

$$
\forall \omega \subset \Gamma_{1, C}, \quad \int_{\omega} \sigma\left(u_{1}\right) n_{1} \mathrm{~d} \Gamma=-\int_{\Pi(\omega)} \sigma\left(u_{2}\right) n_{2} \mathrm{~d} \Gamma=-\int_{\omega} \sigma\left(u_{2} \circ \Pi\right) n_{2} \circ \Pi\left|\operatorname{det}\left(J_{\Pi}\right)\right| \mathrm{d} \Gamma .
$$

These jumps being defined, the unilateral frictionless contact conditions can be expressed on the

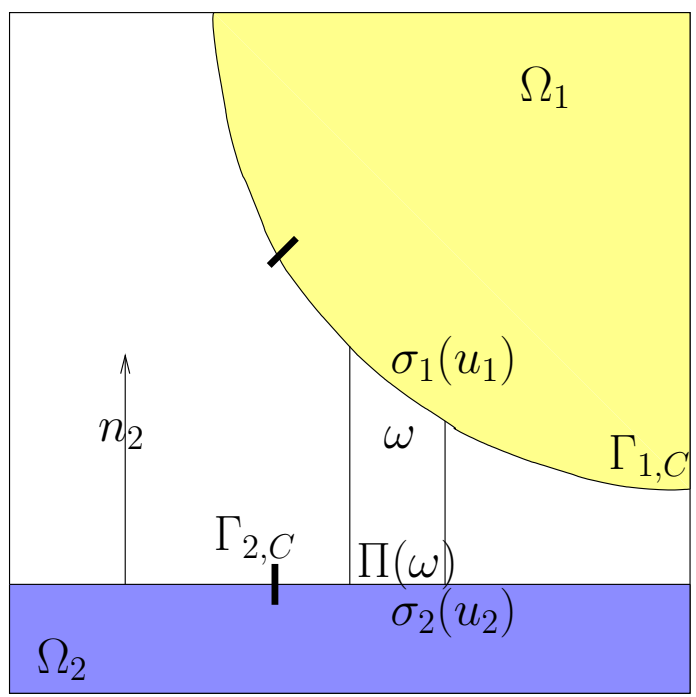

Figure 2: An example illustrating the action-reaction principle between the two bodies.

slave boundary $\Gamma_{1, C}$ as follows:

$$
\left\{\begin{array}{cc}
\llbracket u \cdot n \rrbracket \leqslant g & (i) \\
\sigma_{n}\left(u_{1}\right) \leqslant 0 & (i i), \\
\sigma_{n}\left(u_{1}\right)(\llbracket u \cdot n \rrbracket-g)=0 & (i i i), \\
\llbracket \sigma(u) n \rrbracket=0 & (i v), \\
\sigma_{t}\left(u_{1}\right)=0 & (v) .
\end{array}\right.
$$

Now, let us introduce the Hilbert space $V$ and the convex cone $K$ of admissible displacements:

$$
V=H^{1}\left(\Omega_{1}\right)^{d} \times H^{1}\left(\Omega_{2}\right)^{d},
$$

$K=\left\{v=\left(v_{1}, v_{2}\right) \in V \mid v_{1}=u_{1, D}\right.$ on $\Gamma_{1, D}$ and $v_{2}=u_{2, D}$ on $\Gamma_{2, D} \mid \llbracket v \cdot n \rrbracket-g \leqslant 0$ on $\left.\Gamma_{1, C}\right\}$.

We assume that $f$ belongs to $L^{2}\left(\Omega_{1}\right)^{d} \times L^{2}\left(\Omega_{2}\right)^{d}, \ell$ belongs to $L^{2}\left(\Gamma_{1, N}\right)^{d} \times L^{2}\left(\Gamma_{2, N}\right)^{d}$ and $u_{D}$ belongs to $H^{\frac{3}{2}}\left(\Gamma_{1, D}\right)^{d} \times H^{\frac{3}{2}}\left(\Gamma_{2, D}\right)^{d}$. We define the bilinear and the linear forms $a(.,$.$) and L($. by

$$
a(u, v)=\sum_{i=1,2} \int_{\Omega_{i}} \sigma\left(u_{i}\right): \varepsilon\left(v_{i}\right) \mathrm{d} \Omega, \quad L(v)=\sum_{i=1,2} \int_{\Omega_{i}} f_{i} v_{i} \mathrm{~d} \Omega+\sum_{i=1,2} \int_{\Gamma_{i, N}} \ell_{i} v_{i} \mathrm{~d} \Gamma .
$$


The weak formulation of Problem (1)-(3) as a variational inequality (see [10, 15, 18, 28]), reads:

$$
\left\{\begin{array}{l}
\text { Find } u \in K \text { such that } \\
a(u, v-u) \geqslant L(v-u)
\end{array} \quad \forall v \in K .\right.
$$

Stampacchia's Theorem ensures that Problem (4) admits a unique solution.

\section{A Nitsche-based finite element approximation}

\subsection{Nitsche's formulation}

In this section, we assume that both the solution $u$ and the test functions $v$ are sufficiently regular (for instance, $(u, v) \in\left(H^{3 / 2+\nu}\left(\Omega_{1}\right)^{d} \times H^{3 / 2+\nu}\left(\Omega_{2}\right)^{d}\right)^{2}$ for $\left.\nu>0\right)$. From the equilibrium equations and Green's formula, we obtain:

$$
a(u, v)-\sum_{i=1,2} \int_{\Gamma_{i, D}} \sigma\left(u_{i}\right) n_{i} \cdot v_{i} \mathrm{~d} \Gamma-\int_{\Gamma_{1, C}} \sigma_{n}\left(u_{1}\right) \llbracket v \cdot n \rrbracket \mathrm{d} \Gamma=L(v) .
$$

In order to build Nitsche's formulations for the contact and Dirichlet conditions, the contact conditions are expressed in an equivalent way by extending to our case the formulation given in $[5,6]$. Denoting $z_{+}=\max (z, 0)$ and for an arbitrary $\gamma>0$, the contact conditions $(3)$ on $\Gamma_{1, C}$ can be equivalently rewritten:

$$
\sigma_{n}\left(u_{1}\right)=-\frac{1}{\gamma}\left[\llbracket u \cdot n \rrbracket-g-\gamma \sigma_{n}\left(u_{1}\right)\right]_{+}
$$

Let $\theta \in \mathbb{R}$ be a fixed parameter. This additional parameter for Nitsche's method determines the symmetry properties (see remarks $(3.2)$ and $[5,6]$ ). Then by using (5) and $\llbracket v \cdot n \rrbracket=(\llbracket v \cdot n \rrbracket-$ $\left.\left.\theta \gamma \sigma_{n}(v)\right)+\theta \gamma \sigma_{n}(v)\right)$, we obtain:

$$
\begin{aligned}
& a(u, v)-\int_{\Gamma_{1, C}} \theta \gamma \sigma_{n}\left(u_{1}\right) \sigma_{n}\left(v_{1}\right) \mathrm{d} \Gamma-\sum_{i=1,2} \int_{\Gamma_{i, D}} \sigma\left(u_{i}\right) n_{i} \cdot v_{i} \mathrm{~d} \Gamma \\
& +\int_{\Gamma_{1, C}} \frac{1}{\gamma}\left[\llbracket u \cdot n \rrbracket-g-\gamma \sigma_{n}\left(u_{1}\right)\right]_{+}\left(\llbracket v \cdot n \rrbracket-\theta \gamma \sigma_{n}\left(v_{1}\right)\right) \mathrm{d} \Gamma=L(v) .
\end{aligned}
$$

Using contact conditions (3), it holds $\sigma_{n}\left(u_{1}\right)=\sigma_{n}\left(u_{2} \circ \Pi\right)\left|\operatorname{det}\left(J_{\Pi}\right)\right|$. In order to ensure the stability, we introduce a stabilized formulation for elements having a small contribution $[14,4,16]$. We replace $\sigma_{n}\left(u_{1}\right)$ by a convex combination of $\sigma_{n}\left(u_{1}\right)$ and $\sigma_{n}\left(u_{2} \circ \Pi\right)\left|\operatorname{det}\left(J_{\Pi}\right)\right|$. Namely, we define

$$
\sigma_{n}(u)=t \sigma_{n}\left(u_{2} \circ \Pi\right)\left|\operatorname{det}\left(J_{\Pi}\right)\right|+(1-t) \sigma_{n}\left(u_{1}\right),
$$

for a parameter $t \in[0,1]$ which may be different for an element to an other for the finite element approximation. Note that a similar approach has been developed in [1] where an optimal choice of the fixed parameter $t \in[0,1]$ is proposed. We obtain:

$$
\begin{aligned}
& a(u, v)-\int_{\Gamma_{1, C}} \theta \gamma \sigma_{n}(u) \sigma_{n}(v) \mathrm{d} \Gamma-\sum_{i=1,2} \int_{\Gamma_{i, D}} \sigma_{n}(u) n_{i} \cdot v_{i} \mathrm{~d} \Gamma \\
& +\int_{\Gamma_{1, C}} \frac{1}{\gamma}\left[\llbracket u \cdot n \rrbracket-g-\gamma \sigma_{n}(u)\right]_{+}\left(\llbracket v \cdot n \rrbracket-\theta \gamma \sigma_{n}(v)\right) \mathrm{d} \Gamma=L(v) .
\end{aligned}
$$


We did not treat yet the Dirichlet conditions. In order to be coherent with the fictitious domain approach, we also describe the Dirichlet conditions thanks to Nitsche's method $[14,4,17]$. Then, writing $v_{i}=\left(v_{i}-\theta \gamma \sigma\left(v_{i}\right) n_{i}\right)+\theta \gamma \sigma\left(v_{i}\right) n_{i}$ as in the formulation for the contact conditions, we deduce:

$$
\begin{aligned}
& -\int_{\Gamma_{i, D}} \sigma\left(u_{i}\right) n_{i} \cdot v_{i} \mathrm{~d} \Gamma \\
= & \int_{\Gamma_{i, D}} \frac{1}{\gamma}\left(u_{i}-u_{i, D}-\gamma \sigma\left(u_{i}\right) n_{i}\right) \cdot\left(v_{i}-\gamma \theta \sigma\left(v_{i}\right) n_{i}\right) \mathrm{d} \Gamma-\int_{\Gamma_{i, D}} \theta \gamma \sigma\left(u_{i}\right) n_{i} \cdot \sigma\left(v_{i}\right) n_{i} \mathrm{~d} \Gamma .
\end{aligned}
$$

We obtain the following weak formulation:

$$
\begin{aligned}
& a(u, v)+\int_{\Gamma_{1, C}} \frac{1}{\gamma}\left[\llbracket u \cdot n \rrbracket-g-\gamma \sigma_{n}(u)\right]_{+}\left(\llbracket v \cdot n \rrbracket-\theta \gamma \sigma_{n}(v)\right) \mathrm{d} \Gamma \\
& +\sum_{i=1,2} \int_{\Gamma_{i, D}} \frac{1}{\gamma}\left(u_{i}-u_{i, D}-\gamma \sigma\left(u_{i}\right) n_{i}\right) \cdot\left(v_{i}-\gamma \theta \sigma\left(v_{i}\right) n_{i}\right) \mathrm{d} \Gamma \\
& -\int_{\Gamma_{1, C}} \theta \gamma \sigma_{n}(u) \sigma_{n}(v) \mathrm{d} \Gamma-\sum_{i=1,2} \int_{\Gamma_{i, D}} \theta \gamma \sigma\left(u_{i}\right) n_{i} \cdot \sigma\left(v_{i}\right) n_{i} \mathrm{~d} \Gamma=L(v) \quad \forall v \in V .
\end{aligned}
$$

Finally, defining the bilinear form

$$
A_{\theta \gamma}(u, v)=a(u, v)-\int_{\Gamma_{1, C}} \theta \gamma \sigma_{n}(u) \sigma_{n}(v) \mathrm{d} \Gamma-\sum_{i=1,2} \int_{\Gamma_{i, D}} \theta \gamma \sigma\left(u_{i}\right) n_{i} \cdot \sigma\left(v_{i}\right) n_{i} \mathrm{~d} \Gamma
$$

our Nitsche-based method reads:

$$
\begin{aligned}
& A_{\theta \gamma}(u, v)+\int_{\Gamma_{1, C}} \frac{1}{\gamma}\left[\llbracket u \cdot n \rrbracket-g-\gamma \sigma_{n}(u)\right]_{+}\left(\llbracket v \cdot n \rrbracket-\theta \gamma \sigma_{n}(v)\right) \mathrm{d} \Gamma \\
& +\sum_{i=1,2} \int_{\Gamma_{i, D}} \frac{1}{\gamma}\left(u_{i}-u_{i, D}-\gamma \sigma\left(u_{i}\right) n_{i}\right) \cdot\left(v_{i}-\gamma \theta \sigma\left(v_{i}\right) n_{i}\right) \mathrm{d} \Gamma=L(v) \quad \forall v \in V .
\end{aligned}
$$

\subsection{Discrete Nitsche's formulation}

In what follows, Ciarlet's notations [8] are used. Let $T_{h}$ be a family of triangulations of the fictitious domain $\Omega$ such that $\Omega=\bigcup_{K \in T_{h}} K$. Let $h_{K}$ be the diameter of $K \in T_{h}$ and $h=$ $\max _{K \in T_{h}} h_{K}$. The family of triangulations is assumed to be regular, i.e. it exists $C>0$ such that $\frac{h_{K}}{\rho_{K}} \leqslant C$ where $\rho_{K}$ denotes the radius of the ball inscribed in $K$. We suppose that the mesh is quasi uniform in the sense that it exists $\zeta>0$ a constant such that $\forall K \in T_{h}, h_{K} \geqslant \zeta h$.

Let $\hat{K}$ be the fixed reference element (a triangle for $d=2$, a tetrahedron for $d=3$ ) and let $T_{K}$ be the geometric transformation which satisfies $T_{K}(\hat{K})=K$. The family of triangulations is supposed affine, i.e. $T_{K}$ reads as

$$
\forall K \in T_{h}, T_{K}(\hat{x})=J_{K} \hat{x}+b_{K}, \hat{x} \in \hat{K},
$$

where $J_{K} \in \mathbb{R}^{d, d}$ is the Jacobian matrix of $T_{K}$ being invertible and $b_{K} \in \mathbb{R}^{d}$. Thus, we have:

$$
\left|\operatorname{det}\left(J_{K}\right)\right|=\frac{\operatorname{mes}(K)}{\operatorname{mes}(\hat{K})}, \quad\left\|J_{K}\right\| \leqslant h_{K} / \rho_{\hat{K}}, \quad\left\|J_{K}^{-1}\right\| \leqslant h_{\hat{K}} / \rho_{K} .
$$


Remark 3.1. The family of triangulations is regular and affine, so it holds:

$$
\left|\operatorname{det}\left(J_{K}\right)\right| \leqslant C h_{K}^{d}, \quad\left\|J_{K}\right\| \leqslant C h_{K}, \quad\left\|J_{K}^{-1}\right\| \leqslant C h_{K}^{-1} .
$$

We introduce $U^{h} \subset H^{1}(\Omega)$ a family of finite element spaces indexed by $h$ coming from some order $k \geqslant 1$ finite element method defined on $T_{h}$. Consequently, we suppose the existence of a global interpolation operator $\pi^{h}: \mathscr{C}^{0}(\bar{\Omega}) \rightarrow U^{h}$ and a local one $\pi_{K}^{h}$ on each element $K \in T_{h}$ such that:

$$
\forall u \in \mathscr{C}^{0}(\bar{\Omega}),\left.\quad \pi^{h}(u)\right|_{K}=\pi_{K}^{h}\left(u_{\mid K}\right) \quad \text { and } \quad \forall p \in P_{k}(K), \pi_{K}^{h}(p)=p .
$$

We assume that the finite element method satisfies the following classical local interpolation error estimate for $k \geqslant l \geqslant 0, u \in H^{l+1}(\Omega)$ :

$$
\left\|u-\pi_{K}^{h} u\right\|_{m, K} \leqslant C h^{l+1-m}|u|_{l+1, K}, \quad \text { with } 0 \leqslant m \leqslant l \leqslant k .
$$

Note that, in particular, the classical $P_{k}$ Lagrange finite element method [8] satisfies this estimate. The approximation spaces for our problem are defined by

$$
V_{1}^{h}=\left.\left(U^{h}\right)^{d}\right|_{\Omega_{1}} \quad, \quad V_{2}^{h}=\left.\left(U^{h}\right)^{d}\right|_{\Omega_{2}} \quad \text { and } \quad V^{h}=\left(V_{1}^{h} \times V_{2}^{h}\right) .
$$

In the same way, we define the global operators

$$
\Pi_{i}^{h}: H^{k+1}(\Omega)^{d} \rightarrow V_{i}^{h}, i=\{1,2\} \quad \text { and } \quad \Pi^{h}: H^{k+1}(\Omega)^{d} \times H^{k+1}(\Omega)^{d} \rightarrow V^{h} .
$$

In order to write a discrete approximation of formulation (9), let us introduce the following discrete linear operators:

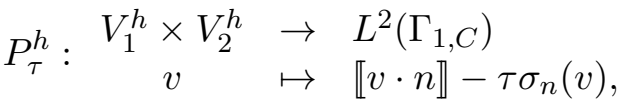

$$
\begin{aligned}
& \bar{P}_{i, \tau}^{h}: \begin{array}{ll}
V_{i}^{h} & \rightarrow L^{2}\left(\Gamma_{i, D}\right)^{d} \\
v_{i} & \mapsto v_{i}-\tau \sigma\left(v_{i}\right) n_{i} .
\end{array}
\end{aligned}
$$

Then, a finite element approximation of our Nitsche-based method reads as:

$$
\left\{\begin{array}{l}
\text { Find } u^{h} \in V^{h} \text { such that } \\
A_{\theta \gamma}\left(u^{h}, v^{h}\right)+\int_{\Gamma_{1, C}} \frac{1}{\gamma}\left[P_{\gamma}^{h}\left(u^{h}\right)-g\right]_{+} P_{\theta \gamma}^{h}\left(v^{h}\right) \mathrm{d} \Gamma \\
+\sum_{i=1,2} \int_{\Gamma_{i, D}} \frac{1}{\gamma}\left(\bar{P}_{i, \gamma}^{h}\left(u_{i}^{h}\right)-u_{i, D}\right) \cdot \bar{P}_{i, \gamma \theta}^{h}\left(v_{i}^{h}\right) \mathrm{d} \Gamma=L\left(v^{h}\right) \quad \forall v^{h} \in V^{h} .
\end{array}\right.
$$

In the following, we define $\gamma=\gamma_{0} h_{K}$.

Remark 3.2. The additional parameter $\theta$ is aimed to be chosen in $[-1,1]$. The following values of $\theta$ are of particular interest: for $\theta=1$, we recover the symmetric method proposed and analyzed in [5]; for $\theta=0$, we recover a non-symmetric version presented in [6] and for $\theta=-1$, we obtain a skew-symmetric version which has the remarkable property that convergence occurs for any value of $\gamma_{0}$ (see [6]).

Remark 3.3. Note that, concerning the Dirichlet conditions, we obtain Nitsche's classical reformulation since the terms on $\Gamma_{i, D}$ in (10) read

$$
\int_{\Gamma_{i, D}} \frac{1}{\gamma}\left(u_{i}-u_{i, D}\right) \cdot v_{i} d \Gamma-\theta \int_{\Gamma_{i, D}}\left(u_{i}-u_{i, D}\right) \cdot \sigma\left(v_{i}\right) n_{i} d \Gamma-\int_{\Gamma_{i, D}} \sigma\left(u_{i}\right) n_{i} \cdot v_{i} d \Gamma .
$$

Indeed, the first term is a kind of penalty term for the Dirichlet condition, the second one ensure the symmetry when $\theta=1$ and the third one ensure the consistency. 


\subsection{Consistency}

The advantage of Nitsche's method, compared to penalization, is the consistency of the approximation in the following sense.

Theorem 3.4. Let $u$ be the solution to Problem (1)-(3). Assume $u$ is sufficiently regular (typically, $\left(u_{1}, u_{2}\right) \in H^{2+\nu}\left(\Omega_{1}\right)^{d} \times H^{2+\nu}\left(\Omega_{2}\right)^{d}$, for $\left.\nu>0\right)$, then $u$ is also a solution to the discrete problem (10) replacing $u^{h}$ by $u$.

Proof. Let $u$ be the solution to (1)-(3) and take $v^{h} \in V^{h}$. We assume $u$ sufficiently regular such that $\sigma_{n}(u) \in L^{2}\left(\Gamma_{1, C}\right)$ and for $i=1,2, \sigma_{n}\left(u_{i}\right) \in L^{2}\left(\Gamma_{i, D}\right)$. As a result, $P_{\theta \gamma}^{h}(u) \in L^{2}\left(\Gamma_{1, C}\right)$, for $i=1,2, \bar{P}_{i, \theta \gamma}^{h}\left(u_{i}\right) \in L^{2}\left(\Gamma_{i, D}\right)$ and $A_{\theta \gamma}\left(u, v^{h}\right)$ makes sense. On the one hand, we use the definition of $P_{\theta \gamma}^{h}, \bar{P}_{i, \theta \gamma}^{h}$, the reformulations (5) and (7) to obtain:

$$
\begin{aligned}
& A_{\theta \gamma}\left(u, v^{h}\right)+\int_{\Gamma_{1, C}} \frac{1}{\gamma}\left[P_{\gamma}^{h}(u)-g\right]_{+} P_{\theta \gamma}^{h}\left(v^{h}\right) \mathrm{d} \Gamma+\sum_{i=1,2} \int_{\Gamma_{i, D}} \frac{1}{\gamma}\left(\bar{P}_{i, \gamma}^{h}\left(u_{i}\right)-u_{i, D}\right) \cdot \bar{P}_{i, \gamma \theta}^{h}\left(v_{i}^{h}\right) \mathrm{d} \Gamma \\
&=\quad a\left(u, v^{h}\right)-\int_{\Gamma_{1, C}} \theta \gamma \sigma_{n}(u) \sigma_{n}\left(v^{h}\right) \mathrm{d} \Gamma-\sum_{i=1,2} \int_{\Gamma_{i, D}} \theta \gamma \sigma\left(u_{i}\right) n_{i} \cdot \sigma\left(v_{i}^{h}\right) n_{i} \mathrm{~d} \Gamma \\
&+\int_{\Gamma_{1, C}} \frac{1}{\gamma}\left[\llbracket u \cdot n \rrbracket-g-\gamma \sigma_{n}(u)\right]_{+}\left(\llbracket v^{h} \cdot n \rrbracket-\theta \gamma \sigma_{n}\left(v^{h}\right)\right) \mathrm{d} \Gamma \\
&+\sum_{i=1,2} \int_{\Gamma_{i, D}} \frac{1}{\gamma}\left(u_{i}-u_{i, D}-\gamma \sigma\left(u_{i}\right) n_{i}\right) \cdot\left(v_{i}^{h}-\gamma \theta \sigma\left(v_{i}^{h}\right) n_{i}\right) \mathrm{d} \Gamma \\
&= a\left(u, v^{h}\right)-\int_{\Gamma_{1, C}} \theta \gamma \sigma_{n}(u) \sigma_{n}\left(v^{h}\right) \mathrm{d} \Gamma+\int_{\Gamma_{1, C}} \frac{1}{\gamma}\left(-\gamma \sigma_{n}(u)\right)\left(\llbracket v^{h} \cdot n \rrbracket-\theta \gamma \sigma_{n}\left(v^{h}\right)\right) \mathrm{d} \Gamma \\
&+\sum_{i=1,2} \int_{\Gamma_{i, D}} \frac{1}{\gamma}\left(-\gamma \sigma\left(u_{i}\right) n_{i}\right) \cdot\left(v_{i}^{h}-\gamma \theta \sigma\left(v_{i}^{h}\right) n_{i}\right) \mathrm{d} \Gamma-\sum_{i=1,2} \int_{\Gamma_{i, D}} \theta \gamma \sigma\left(u_{i}\right) n_{i} \cdot \sigma\left(v_{i}^{h}\right) n_{i} \mathrm{~d} \Gamma \\
&= a\left(u, v^{h}\right)-\int_{\Gamma_{1, C}} \sigma_{n}(u) \llbracket v^{h} \cdot n \rrbracket \mathrm{d} \Gamma-\sum_{i=1,2} \int_{\Gamma_{i, D}} \sigma\left(u_{i}\right) n_{i} \cdot v_{i}^{h} \mathrm{~d} \Gamma \\
&= a\left(u, v^{h}\right)-\int_{\Gamma_{1, C}} \sigma_{n}\left(u_{1}\right) \llbracket v^{h} \cdot n \rrbracket \mathrm{d} \Gamma-\sum_{i=1,2} \int_{\Gamma_{i, D}} \sigma\left(u_{i}\right) n_{i} \cdot v_{i}^{h} \mathrm{~d} \Gamma
\end{aligned}
$$

On the other hand, multiplying by $v_{i}^{h}$ and integrating (1), it holds:

$$
-\sum_{i=1,2} \int_{\Omega_{i}} \operatorname{div} \sigma\left(u_{i}\right) v_{i}^{h} \mathrm{~d} \Omega=\sum_{i=1,2} \int_{\Omega_{i}} f_{i} v_{i}^{h} \mathrm{~d} \Omega .
$$

Using Green's formula, we have:

$$
-\int_{\Omega_{i}} \operatorname{div} \sigma\left(u_{i}\right) v_{i}^{h} \mathrm{~d} \Omega=\int_{\Omega_{i}} \sigma\left(u_{i}\right): \varepsilon\left(v_{i}^{h}\right) \mathrm{d} \Omega-\int_{\Gamma_{i}} \sigma\left(u_{i}\right) n_{i} \cdot v_{i}^{h} \mathrm{~d} \Gamma \quad i=1,2,
$$

with

$$
\begin{aligned}
& -\int_{\Gamma_{i}} \sigma\left(u_{i}\right) n_{i} \cdot v_{i}^{h} \mathrm{~d} \Gamma=-\int_{\Gamma_{i, D}} \sigma\left(u_{i}\right) n_{i} \cdot v_{i}^{h} \mathrm{~d} \Gamma-\int_{\Gamma_{i, N}} \sigma\left(u_{i}\right) n_{i} \cdot v_{i}^{h} \mathrm{~d} \Gamma-\int_{\Gamma_{i, C}} \sigma\left(u_{i}\right) n_{i} \cdot v_{i}^{h} \mathrm{~d} \Gamma \quad i=1,2, \\
& -\int_{\Gamma_{i}} \sigma\left(u_{i}\right) n_{i} \cdot v_{i}^{h} \mathrm{~d} \Gamma=-\int_{\Gamma_{i, D}} \sigma\left(u_{i}\right) n_{i} \cdot v_{i}^{h} \mathrm{~d} \Gamma-\int_{\Gamma_{i, N}} \ell_{i} v_{i}^{h} \mathrm{~d} \Gamma-\int_{\Gamma_{i, C}} \sigma\left(u_{i}\right) n_{i} \cdot v_{i}^{h} \mathrm{~d} \Gamma \quad i=1,2 .
\end{aligned}
$$


Using the one to one correspondence of the projection, it holds:

$$
\int_{\Gamma_{2, C}} \sigma\left(u_{2}\right) n_{2} \cdot v_{2}^{h} \mathrm{~d} \Gamma=\int_{\Gamma_{1, C}} \sigma\left(u_{2} \circ \Pi\right) n_{2} \circ \Pi \cdot v_{2}^{h} \circ \Pi\left|\operatorname{det}\left(J_{\Pi}\right)\right| \mathrm{d} \Gamma .
$$

Hence

$$
\begin{aligned}
-\sum_{i=1,2} \int_{\Omega_{i}} \operatorname{div} \sigma\left(u_{i}\right) v_{i}^{h} \mathrm{~d} \Omega= & \int_{\Omega_{1}} \sigma\left(u_{1}\right): \varepsilon\left(v_{1}^{h}\right) \mathrm{d} \Omega+\int_{\Omega_{2}} \sigma\left(u_{2}\right): \varepsilon\left(v_{2}^{h}\right) \mathrm{d} \Omega-\int_{\Gamma_{1, C}} \sigma\left(u_{1}\right) n_{1} \cdot v_{1}^{h} \mathrm{~d} \Gamma \\
& -\int_{\Gamma_{1, C}} \sigma\left(u_{2} \circ \Pi\right) n_{2} \circ \Pi \cdot v_{2}^{h} \circ \Pi\left|\operatorname{det}\left(J_{\Pi}\right)\right| \mathrm{d} \Gamma \\
& -\sum_{i=1,2} \int_{\Gamma_{i, D}} \sigma\left(u_{i}\right) n_{i} \cdot v_{i}^{h} \mathrm{~d} \Gamma-\int_{\Gamma_{1, N}} \ell_{1} v_{1}^{h} \mathrm{~d} \Gamma-\int_{\Gamma_{2, N}} \ell_{2} v_{2}^{h} \mathrm{~d} \Gamma .
\end{aligned}
$$

Using (3), it holds:

$$
\begin{aligned}
-\sum_{i=1,2} \int_{\Omega_{i}} \operatorname{div} \sigma\left(u_{i}\right) v_{i}^{h} \mathrm{~d} \Omega= & a\left(u, v^{h}\right)-\sum_{i=1,2} \int_{\Gamma_{i, D}} \sigma\left(u_{i}\right) n_{i} \cdot v_{i}^{h} \mathrm{~d} \Gamma-\int_{\Gamma_{1, N}} \ell_{1} v_{1}^{h} \mathrm{~d} \Gamma-\int_{\Gamma_{2, N}} \ell_{2} v_{2}^{h} \mathrm{~d} \Gamma \\
& -\int_{\Gamma_{1, C}} \sigma_{n}\left(u_{1}\right) \llbracket v^{h} \cdot n \rrbracket \mathrm{d} \Gamma .
\end{aligned}
$$

So

$$
a\left(u, v^{h}\right)-\int_{\Gamma_{1, C}} \sigma_{n}\left(u_{1}\right) \llbracket v^{h} \cdot n \rrbracket \mathrm{d} \Gamma-\sum_{i=1,2} \int_{\Gamma_{i, D}} \sigma\left(u_{i}\right) n_{i} \cdot v_{i}^{h} \mathrm{~d} \Gamma=L\left(v^{h}\right) .
$$

Which ends the proof.

Moreover, formulation (9) is formally equivalent to (1) and (3) in the following sense.

Theorem 3.5. Let $u \in H^{2}\left(\Omega_{1}\right)^{d} \times H^{2}\left(\Omega_{2}\right)^{d}$ be a solution to equation (9) then $u$ is a solution to (1) and (3).

Proof. For $u \in H^{2}\left(\Omega_{1}\right)^{d} \times H^{2}\left(\Omega_{2}\right)^{d}$ a solution to (9) and whatever $v \in H^{2}\left(\Omega_{1}\right)^{d} \times H^{2}\left(\Omega_{2}\right)^{d}$, it satisfies:

$$
\int_{\Omega_{i}}\left(\operatorname{div} \sigma\left(u_{i}\right)+f_{i}\right) v_{i} \mathrm{~d} \Omega=0 \quad \forall v_{i} \in H^{2}\left(\Omega_{i}\right)
$$

i.e.

$$
-\operatorname{div} \sigma\left(u_{i}\right)=f_{i} \quad \text { a.e. in } \Omega_{i}, 1 \leqslant i \leqslant 2 .
$$

We have, for all $v \in H^{2}\left(\Omega_{1}\right)^{d} \times H^{2}\left(\Omega_{2}\right)^{d}$ :

$$
\begin{aligned}
\int_{\Gamma_{1, C}} \frac{1}{\gamma}\left[\llbracket u \cdot n \rrbracket-g-\gamma \sigma_{n}(u)\right]_{+} \llbracket v \cdot n \rrbracket \mathrm{d} \Gamma+\int_{\Gamma_{1, C}} \sigma\left(u_{1}\right) n_{1} \cdot v_{1} \mathrm{~d} \Gamma+\int_{\Gamma_{2, C}} \sigma\left(u_{2}\right) n_{2} \cdot v_{2} \mathrm{~d} \Gamma=0, \\
\quad \int_{\Gamma_{1, C}} \frac{1}{\gamma}\left[\llbracket u \cdot n \rrbracket-g-\gamma \sigma_{n}(u)\right]_{+}\left(v_{1}-v_{2} \circ \Pi \cdot n\right) \mathrm{d} \Gamma-\int_{\Gamma_{1, C}} \sigma_{n}\left(u_{1}\right) n \cdot v_{1} \mathrm{~d} \Gamma \\
\quad+\int_{\Gamma_{1, C}} \sigma_{n}\left(u_{2} \circ \Pi\right) n \cdot v_{2} \circ \Pi\left|\operatorname{det}\left(J_{\Pi}\right)\right| \mathrm{d} \Gamma=0 .
\end{aligned}
$$


Hence

$$
\int_{\Gamma_{1, C}}\left(\frac{1}{\gamma}\left[\llbracket u \cdot n \rrbracket-g-\gamma \sigma_{n}(u)\right]_{+}+\sigma_{n}\left(u_{1}\right)\right) v_{1} \cdot n \mathrm{~d} \Gamma=0 \forall v_{1} \in H^{2}\left(\Omega_{1}\right)
$$

and

$$
\int_{\Gamma_{1, C}}\left(\frac{1}{\gamma}\left[\llbracket u \cdot n \rrbracket-g-\gamma \sigma_{n}(u)\right]_{+}-\sigma_{n}\left(u_{2} \circ \Pi\right)\left|\operatorname{det}\left(J_{\Pi}\right)\right|\right) v_{2} \circ \Pi \cdot n \mathrm{~d} \Gamma=0 \forall v_{2} \in H^{2}\left(\Omega_{2}\right) .
$$

Hence

$$
\frac{1}{\gamma}\left[\llbracket u \cdot n \rrbracket-g-\gamma \sigma_{n}(u)\right]_{+}=-\sigma_{n}\left(u_{1}\right) \quad \text { a. e. on } \Omega_{1},
$$

which is a formulation equivalent to (3). Arguing in the same way as above the Neumann and Dirichlet conditions are recovered.

\subsection{Stabilization method}

A stabilization technique is necessary to control the possible bad quality of $\sigma_{n}\left(u^{h}\right)$ on elements having very small intersection with the real domains. The stabilization used is the one proposed in [16] which consists in using extension of the normal stress on a neighbor element having a sufficiently large intersection with the real domain. The advantage of this stabilization technique is the absence of parameter to fit, except the threshold under which an intersection is considered to be too small. Note that other stabilization techniques are available, such as the so-called ghost penalty stabilization considered in [4].

For a given small radius $1>\hat{\rho}>0$, let $R_{\hat{\rho}}$ (respectively $\bar{R}_{\hat{\rho}}$ ) be an operator of approximation of the normal stress of displacements $\sigma_{n}\left(u^{h}\right)$ (respectively $\sigma\left(u_{i}^{h}\right)$ ) which we define thereafter. For $K \in T_{h}$ such that $K \cap \Gamma_{1, C}$, we note $S_{K}=\left\{K^{\prime} \in T_{h} \mid K^{\prime} \cap \Pi(K) \neq \emptyset\right\}$. We note also $E_{K}$, the polynomial extrapolation of an element $v^{h} \in V^{h}$ define from $K$ to $\Omega$.

We distinguish three cases to define the stabilized operator $R_{\hat{\rho}}$. Let $K \in T_{h}$ and $K \cap \Gamma_{1, C} \neq \emptyset$ then:

- if the intersection between $K$ and $\Omega_{1}$ is sufficiently large i.e. it exists $\hat{y}_{K}>0$ such that $B\left(\hat{y}_{K}, \hat{\rho}\right) \subset T_{K}^{-1}\left(K \cap \Omega_{1}\right)$ (see Figure $\left.3 a\right)$ ), then $\left.R_{\hat{\rho}}\left(v^{h}\right)\right|_{K}=\sigma_{n}\left(\left.v_{1}^{h}\right|_{K}\right)$,

- otherwise, if it exists $\widetilde{K} \in S_{K}$ intersecting $\Omega_{2}$ such that it exists $\hat{y}_{\widetilde{K}}>0$ with $B\left(\hat{y}_{\widetilde{K}}, \hat{\rho}\right) \subset$ $T_{\widetilde{K}}^{-1}\left(\widetilde{K} \cap \Omega_{2}\right)$ (see Figure $\left.3 b\right)$ ), then $\left.R_{\hat{\rho}}\left(v^{h}\right)\right|_{K}=\sigma_{n}\left(E_{\widetilde{K}}\left(v_{2}^{h}\right) \circ \Pi\right)\left|\operatorname{det}\left(J_{\Pi}\right)\right|$,

- otherwise, we suppose that it exits a neighbor element $K^{\prime}$ of $K$ such that it exists $\hat{y}_{K^{\prime}}>0$ with $B\left(\hat{y}_{K^{\prime}}, \hat{\rho}\right) \subset T_{K^{\prime}}^{-1}\left(K^{\prime} \cap \Omega_{1}\right)$ (see Figure $\left.3 c\right)$ ), then $\left.R_{\hat{\rho}}\left(v^{h}\right)\right|_{K}=\sigma_{n}\left(E_{K^{\prime}}\left(v_{1}^{h}\right)\right.$ ).

In the same way, we define the operator $\bar{R}_{\hat{\rho}}$ on $\Gamma_{i, D}$ for $i=1,2$ :

$$
\begin{aligned}
\bar{R}_{\hat{\rho}}: & \rightarrow L^{2}\left(\Gamma_{i, D}\right)^{d} \\
v_{i} & \mapsto \bar{R}_{\hat{\rho}}\left(v_{i}^{h}\right)=\left\{\begin{array}{l}
\sigma\left(v_{i}^{h}\right) n_{i} \quad \exists \hat{y}_{K}>0 \text { such that } B\left(\hat{y}_{K}, \hat{\rho}\right) \subset T_{K}^{-1}\left(K \cap \Omega_{1}\right) \\
\sigma\left(E_{K^{\prime}}\left(v_{i}^{h}\right)\right) n_{i} \quad \text { otherwise. }
\end{array}\right.
\end{aligned}
$$

Let us introduce the stabilized discrete linear operators:

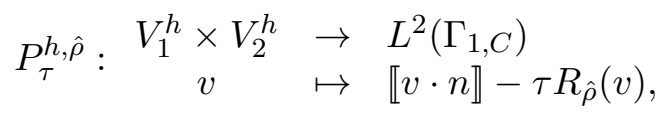




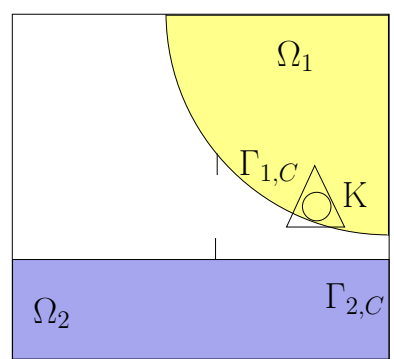

a) If $\Omega_{1} \cap K$ is sufficiently large

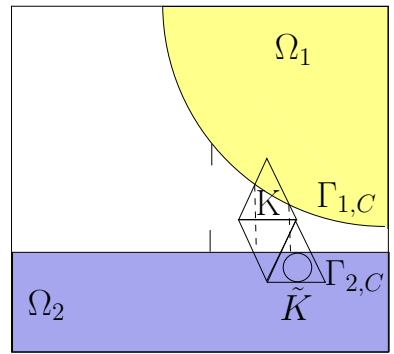

b) If $\exists \tilde{K} \in S_{K}$ such that $\Omega_{2} \cap \tilde{K}$ is sufficiently large

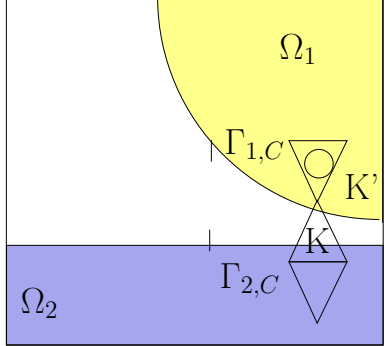

c) Otherwise

Figure 3: The different cases for the definition of $R_{\hat{\rho}}$.

$$
\bar{P}_{i, \tau}^{h, \hat{\rho}}: \begin{aligned}
& V_{i}^{h} \rightarrow L^{2}\left(\Gamma_{i, D}\right)^{d} \\
& v_{i} \mapsto v_{i}-\tau \bar{R}_{\hat{\rho}}\left(v_{i}\right) .
\end{aligned}
$$

We define the discrete form of $A_{\theta \gamma}(.,$.$) as follows:$

$$
A_{\theta \gamma}^{h}\left(u^{h}, v^{h}\right)=a\left(u^{h}, v^{h}\right)-\int_{\Gamma_{1, C}} \theta \gamma R_{\hat{\rho}}\left(u^{h}\right) R_{\hat{\rho}}\left(v^{h}\right) \mathrm{d} \Gamma-\sum_{i=1,2} \int_{\Gamma_{i, D}} \theta \gamma \bar{R}_{\hat{\rho}}\left(u_{i}^{h}\right) \bar{R}_{\hat{\rho}}\left(v_{i}^{h}\right) \mathrm{d} \Gamma .
$$

The stabilized version of our approximation (10) reads:

$$
\left\{\begin{array}{l}
\text { Find } u^{h} \in V^{h} \text { such that } \\
A_{\theta \gamma}^{h}\left(u^{h}, v^{h}\right)+\int_{\Gamma_{1, C}} \frac{1}{\gamma}\left[P_{\gamma}^{h, \hat{\rho}}\left(u^{h}\right)-g\right]_{+} P_{\theta \gamma}^{h, \hat{\rho}}\left(v^{h}\right) \mathrm{d} \Gamma \\
+\sum_{i=1,2} \int_{\Gamma_{i, D}} \frac{1}{\gamma}\left(\bar{P}_{i, \gamma}^{h, \hat{\rho}}\left(u_{i}^{h}\right)-u_{i, D}\right) \cdot \bar{P}_{i, \gamma \theta}^{h, \hat{\rho}}\left(v_{i}^{h}\right) \mathrm{d} \Gamma=L\left(v^{h}\right) \quad \forall v^{h} \in V^{h} .
\end{array}\right.
$$

Note that strict consistency of this stabilized discrete problem do not occur. However, we have the following result.

Theorem 3.6. Let $u$ be the solution to Problem (1)-(3). Assume $u$ is sufficiently regular (typically, $\left(u_{1}, u_{2}\right) \in H^{2+\nu}\left(\Omega_{1}\right)^{d} \times H^{2+\nu}\left(\Omega_{2}\right)^{d}$ for $\left.\nu>0\right)$, then $u$ is also a solution to the following problem:

$$
\left\{\begin{array}{l}
a\left(u, v^{h}\right)-\int_{\Gamma_{1, C}} \theta \gamma \sigma_{n}(u) R_{\hat{\rho}}\left(v^{h}\right) d \Gamma-\sum_{i=1,2} \int_{\Gamma_{i, D}} \theta \gamma \sigma\left(u_{i}\right) n_{i} \cdot \bar{R}_{\hat{\rho}}\left(v_{i}^{h}\right) d \Gamma \\
+\int_{\Gamma_{1, C}} \frac{1}{\gamma}\left[P_{\gamma}^{h}(u)-g\right]_{+} P_{\theta \gamma}^{h, \hat{\rho}}\left(v^{h}\right) d \Gamma \\
+\sum_{i=1,2} \int_{\Gamma_{i, D}} \frac{1}{\gamma}\left(\bar{P}_{i, \gamma}^{h}\left(u_{i}\right)-u_{i, D}\right) \cdot \bar{P}_{i, \gamma \theta}^{h, \hat{\rho}}\left(v_{i}^{h}\right) d \Gamma=L\left(v^{h}\right) \quad \forall v^{h} \in V^{h} .
\end{array}\right.
$$

Proof. The proof can be straightforwardly deduced from the one of Theorem 3.4.

\section{Analysis of the Nitsche-based approximation}

\subsection{Existence and uniqueness results}

Theorem 4.1. Let $\gamma=\gamma_{0} h_{K}$. It exists a unique solution $v^{h} \in V^{h}$ to the discrete problem (11), for all $\gamma_{0}>0$ if $\theta=-1$ and for $\gamma_{0}>0$ sufficiently small if $\theta \neq-1$. 
Proof. The proof is adapted from [6]. The main adaptations concern the fictitious domain framework and in particular the stabilization term, the consideration of two elastic solids and the semi-coercivity of the bilinear form due to the fact that Dirichlet conditions are taken into account with Nitsche's method. We begin by providing some stability and approximation property for operators $R_{\hat{\rho}}$ and $\bar{R}_{\hat{\rho}}$ in lemmas 4.2, 4.5 and 4.6. Then a coercivity property is proved in Lemma 4.7. Finally, the existence and uniqueness result is deduce from the hemi-continuity of the nonlinear operator which corresponds to (11).

Lemma 4.2. Let $v^{h} \in V^{h}$, there exists a constant $C>0$ independent of $h$ such that

$$
\left\|R_{\hat{\rho}}\left(v^{h}\right)\right\|_{0, \Gamma_{1, C}}^{2} \leqslant C h^{-1}\left(\left\|v_{1}^{h}\right\|_{1, \Omega_{1}}^{2}+\left\|v_{2}^{h}\right\|_{1, \Omega_{2}}^{2}\right) \quad \forall v^{h} \in V^{h} .
$$

The proof of this lemma is detailed in the appendix.

Remark 4.3. The following more general operator $R_{\hat{\rho}}$ could be considered:

$$
\left.R_{\hat{\rho}}\left(u^{h}\right)\right|_{K}=(1-t) \sigma_{n}\left(E_{K^{\prime}}\left(u_{2}^{h} \circ \Pi\right)\right)\left|\operatorname{det}\left(J_{\Pi}\right)\right|+t \sigma_{n}\left(E_{K^{\prime \prime}}\left(u_{1}^{h}\right)\right),
$$

with $t \in[0,1]$, the element $K^{\prime}$ being $K$ itself or a neighbor element such as the intersection between $K^{\prime}$ and $\Omega_{2}$ is large enough and the element $K^{\prime \prime}$ being $K$ itself or a neighbor element such as the intersection between $K^{\prime \prime}$ and $\Omega_{1}$ is large enough. Lemma 4.2 can be easily extended to this operator. When the elastic coefficients in $\Omega_{1}$ and $\Omega_{2}$ are equal, a proposed optimum choice is given by (see [1]):

$$
t_{K}=\frac{\operatorname{mes}\left(\Omega_{1} \cap K\right)}{m e s\left(\Omega_{1} \cap K\right)+\operatorname{mes}\left(\Omega_{2} \cap K\right)} .
$$

Remark 4.4. When the initial gap between the two bodies vanishes, for $\hat{\rho}$ sufficiently small either $K \cap \Omega_{1}$ or $K \cap \Omega_{2}$ is sufficiently large and thus it is not necessary to consider any neighbor element.

Lemma 4.5. Let $u^{h} \in V^{h}, \Gamma_{i, D}$ be Lipschitz continuous then it exits a constant $C>0$ independent of $h$ such that

$$
\left\|\bar{R}_{\hat{\rho}}\left(u_{1}^{h}\right)\right\|_{0, \Gamma_{1, D}}^{2} \leqslant C h^{-1}\left\|u_{1}^{h}\right\|_{1, \Omega_{1}}^{2},
$$

and

$$
\left\|\bar{R}_{\hat{\rho}}\left(u_{2}^{h}\right)\right\|_{0, \Gamma_{2, D}}^{2} \leqslant C h^{-1}\left\|u_{2}^{h}\right\|_{1, \Omega_{2}}^{2} .
$$

The proof of this lemma can be straightforwardly deduced from the one of Lemma 4.2.

Now, Let $u^{h}, v^{h} \in V^{h}$ and $\gamma=h_{K} \gamma_{0}$ and using lemma 4.2, it holds:

$$
\begin{gathered}
\left\|\gamma^{\frac{1}{2}} R_{\hat{\rho}}\left(u^{h}-v^{h}\right)\right\|_{0, \Gamma_{1, C}}^{2} \leqslant C \gamma_{0} \sum_{i=1,2}\left\|u_{i}^{h}-v_{i}^{h}\right\|_{1, \Omega_{i}}^{2}, \\
\left\|\gamma^{\frac{1}{2}} \bar{R}_{\hat{\rho}}\left(u_{i}^{h}-v_{i}^{h}\right)\right\|_{0, \Gamma_{i, D}}^{2} \leqslant C \gamma_{0}\left\|u_{i}^{h}-v_{i}^{h}\right\|_{1, \Omega_{i}}^{2} .
\end{gathered}
$$

Due to the know approximation properties of the stabilized operators on regular and quasi-uniform families of meshes (see [16]), one obtains the following lemma: 
Lemma 4.6. For any $v \in H^{k+1}\left(\Omega_{1}\right)^{d} \times H^{k+1}\left(\Omega_{2}\right)^{d}$

$$
\left\|R_{\hat{\rho}}\left(\Pi^{h}(v)\right)-\sigma_{n}(v)\right\|_{0, \Gamma_{1, C}}^{2} \leqslant C h^{2 k-1} \sum_{i=1,2}\|v\|_{k+1, \Omega_{i}}^{2},
$$

and

$$
\sum_{i=1,2}\left\|\bar{R}_{\hat{\rho}}\left(\Pi_{i}^{h}\left(v_{i}\right)\right)-\sigma\left(v_{i}\right) n_{i}\right\|_{0, \Gamma_{i, D}}^{2} \leqslant C h^{2 k-1} \sum_{i=1,2}\|v\|_{k+1, \Omega_{i}}^{2}
$$

The following coercivity property can be stated :

Lemma 4.7. For $M>0$ fixed, it exists $\alpha>0$ such that for all $\gamma$ with $M \geqslant \gamma>0$ the following coercivity property holds:

$$
a(v, v)+\frac{1}{2} \sum_{i=1,2} \int_{\Gamma_{i, D}} \gamma^{-1} v_{i}^{2} d \Gamma \geqslant \alpha \sum_{i=1,2}\left\|v_{i}\right\|_{1, \Omega_{i}}^{2} \quad \forall v \in V .
$$

The proof of this lemma is detailed in the appendix. Now, by defining the following operator $B^{h}$ from $V^{h}$ to $V^{h}$ :

$$
\begin{aligned}
\left(B^{h} u^{h}, v^{h}\right)_{1, \Omega}= & A_{\theta \gamma}^{h}\left(u^{h}, v^{h}\right)+\sum_{i=1,2} \int_{\Gamma_{i, D}} \frac{1}{\gamma}\left(\bar{P}_{i, \gamma}^{h, \hat{\rho}}\left(u_{i}^{h}\right)-u_{i, D}\right) \cdot \bar{P}_{i, \gamma \theta}^{h, \hat{\rho}}\left(v_{i}^{h}\right) \mathrm{d} \Gamma \\
& +\int_{\Gamma_{1, C}} \frac{1}{\gamma}\left[P_{\gamma}^{h, \hat{\rho}}\left(u^{h}\right)-g\right]_{+} P_{\theta \gamma}^{h, \hat{\rho}}\left(v^{h}\right) \mathrm{d} \Gamma \quad \forall u^{h}, v^{h} \in V^{h},
\end{aligned}
$$

it is sufficient to prove that $B^{h}$ is hemi-continuous (see the Corollary 15 p. 126 of [3]) to end the proof of Theorem 4.1. The proof of hemi-continuity of $B^{h}$ is detailed in the appendix.

\subsection{A priori Error analysis}

In this section some optimal a priori error estimates are proved for the problem under consideration. The rate of convergence is the same as for standard finite element methods.

Theorem 4.8. Let $u$ be a solution of the stabilized problem (4) belonging to $H^{\frac{3}{2}+\nu}\left(\Omega_{1}\right)^{d} \times$ $H^{\frac{3}{2}+\nu}\left(\Omega_{2}\right)^{d}$ with $\nu>0$.

1. If $\theta \neq-1$, we suppose $\gamma_{0}>0$ is sufficiently small. The solution $u^{h}$ of the stabilized problem (11) satisfies the following error estimate:

$$
\begin{aligned}
& \sum_{i=1,2}\left\|u_{i}-u_{i}^{h}\right\|_{1, \Omega_{i}}^{2}+\left\|\gamma^{\frac{1}{2}}\left(\sigma_{n}(u)+\frac{1}{\gamma}\left[P_{\gamma}^{h, \hat{\rho}}\left(u^{h}\right)-g\right]_{+}\right)\right\|_{0, \Gamma_{1, C}}^{2} \\
& +\sum_{i=1,2}\left\|\gamma^{-\frac{1}{2}}\left(\bar{P}_{i, \gamma}^{h}\left(u_{i}\right)-\bar{P}_{i, \gamma}^{h, \hat{\rho}}\left(u_{i}\right)\right)\right\|_{0, \Gamma_{i, D}}^{2} \\
\leqslant & C \inf _{v^{h} \in V^{h}}\left(\sum_{i=1,2}\left\|u_{i}-v_{i}^{h}\right\|_{1, \Omega_{i}}^{2}+\left\|\gamma^{-\frac{1}{2}}\left(u-v^{h}\right)\right\|_{0, \Gamma_{1, C}}^{2}+\left\|\gamma^{\frac{1}{2}}\left(\sigma_{n}(u)-R_{\hat{\rho}}\left(v^{h}\right)\right)\right\|_{0, \Gamma_{1, C}}^{2}\right. \\
& \left.+\sum_{i=1,2}\left\|\gamma^{-\frac{1}{2}}\left(u_{i}-v_{i}^{h}\right)\right\|_{0, \Gamma_{i, D}}^{2}+\sum_{i=1,2}\left\|\gamma^{\frac{1}{2}}\left(\sigma\left(u_{i}\right)-\bar{R}_{\hat{\rho}}\left(v_{i}^{h}\right)\right)\right\|_{0, \Gamma_{i, D}}^{2}\right)
\end{aligned}
$$

with $C>0$ a constant independent of $h, u$ and $\gamma_{0}$. 
2. If $\theta=-1$, then for all $\gamma_{0}>0$, the solution $u^{h}$ of the stabilized problem (11) satisfies the error estimate (16) with $C>0$ a constant independent of $h$ and $u$.

Proof. The proof is also an adaptation to our fictitious domain framework of the one in [6]. Let $v^{h} \in V^{h}$, using the coercivity inequality (14) and continuity of the form $a(.,)+$. $\frac{1}{2} \sum_{i=1,2} \int_{\Gamma_{i, D}} \gamma^{-1}(.)^{2} \mathrm{~d} \Gamma$ and Young's inequality, it holds:

$$
\begin{aligned}
\alpha \sum_{i=1,2}\left\|u_{i}-u_{i}^{h}\right\|_{1, \Omega_{i}}^{2} \leqslant & a\left(u-u^{h}, u-u^{h}\right)+\frac{1}{2} \sum_{i=1,2} \int_{\Gamma_{i, D}} \gamma^{-1}\left(u_{i}-u_{i}^{h}\right)^{2} \mathrm{~d} \Gamma \\
= & a\left(u-u^{h},\left(u-v^{h}\right)+\left(v^{h}-u^{h}\right)\right)+\frac{1}{2} \sum_{i=1,2}\left\|\gamma^{-\frac{1}{2}}\left(u_{i}-u_{i}^{h}\right)\right\|_{0, \Gamma_{i, D}}^{2} \\
\leqslant & C \sum_{i=1,2}\left\|u_{i}-u_{i}^{h}\right\|_{1, \Omega_{i}}\left\|u_{i}-v_{i}^{h}\right\|_{1, \Omega_{i}}+a\left(u-u^{h}, v^{h}-u^{h}\right) \\
& +\frac{1}{2} \sum_{i=1,2}\left\|\gamma^{-\frac{1}{2}}\left(u_{i}-u_{i}^{h}\right)\right\|_{0, \Gamma_{i, D}}^{2} \\
\leqslant & \frac{\alpha}{2} \sum_{i=1,2}\left\|u_{i}-u_{i}^{h}\right\|_{1, \Omega_{i}}^{2}+\frac{C^{2}}{2 \alpha} \sum_{i=1,2}\left\|u_{i}-v_{i}^{h}\right\|_{1, \Omega_{i}}^{2} \\
& +\frac{1}{2} \sum_{i=1,2}\left\|\gamma^{-\frac{1}{2}}\left(u_{i}-u_{i}^{h}\right)\right\|_{0, \Gamma_{i, D}}^{2}+a\left(u, v^{h}-u^{h}\right)-a\left(u^{h}, v^{h}-u^{h}\right) .
\end{aligned}
$$

Hence

$$
\begin{aligned}
\frac{\alpha}{2} \sum_{i=1,2}\left\|u_{i}-u_{i}^{h}\right\|_{1, \Omega_{i}}^{2} \leqslant & \frac{C^{2}}{2 \alpha} \sum_{i=1,2}\left\|u_{i}-v_{i}^{h}\right\|_{1, \Omega_{i}}^{2}+\frac{1}{2} \sum_{i=1,2}\left\|\gamma^{-\frac{1}{2}}\left(u_{i}-u_{i}^{h}\right)\right\|_{0, \Gamma_{i, D}}^{2} \\
& +a\left(u, v^{h}-u^{h}\right)-a\left(u^{h}, v^{h}-u^{h}\right) .
\end{aligned}
$$

Let $u$ be the solution to (4), it verifies the stabilized formulation (12), thus we have:

$$
\begin{aligned}
a\left(u, v^{h}-u^{h}\right)-a\left(u^{h}, v^{h}-u^{h}\right)= & \int_{\Gamma_{1, C}} \theta \gamma\left(\sigma_{n}(u)-R_{\hat{\rho}}\left(u^{h}\right)\right) R_{\hat{\rho}}\left(v^{h}-u^{h}\right) \mathrm{d} \Gamma \\
& -\sum_{i=1,2} \int_{\Gamma_{i, D}} \frac{1}{\gamma}\left(\bar{P}_{i, \gamma}^{h}\left(u_{i}\right)-\bar{P}_{i, \gamma}^{h, \hat{\rho}}\left(u_{i}^{h}\right)\right) \cdot \bar{P}_{i, \gamma}^{h, \hat{\rho}}\left(v_{i}^{h}-u_{i}^{h}\right) \mathrm{d} \Gamma \\
& +\sum_{i=1,2} \int_{\Gamma_{i, D}} \theta \gamma\left(\sigma\left(u_{i}\right) n_{i}-\bar{R}_{\hat{\rho}}\left(u_{i}^{h}\right)\right) \cdot \bar{R}_{\hat{\rho}}\left(v_{i}^{h}-u_{i}^{h}\right) \mathrm{d} \Gamma \\
& +\int_{\Gamma_{1, C}} \frac{1}{\gamma}\left(\left[P_{\gamma}^{h, \hat{\rho}}\left(u^{h}\right)-g\right]_{+}-\left[P_{\gamma}^{h}(u)-g\right]_{+}\right) P_{\theta \gamma}^{h, \hat{\rho}}\left(v^{h}-u^{h}\right) \mathrm{d} \Gamma .
\end{aligned}
$$

First, using Cauchy-Schwarz and Young's inequalities for $\beta_{1}>0$, it holds:

$$
\begin{aligned}
& \int_{\Gamma_{1, C}} \theta \gamma\left(\sigma_{n}(u)-R_{\hat{\rho}}\left(u^{h}\right)\right) R_{\hat{\rho}}\left(v^{h}-u^{h}\right) \mathrm{d} \Gamma \\
\leqslant & \frac{\theta^{2}}{2 \beta_{1}}\left\|\gamma^{\frac{1}{2}}\left(\sigma_{n}(u)-R_{\hat{\rho}}\left(v^{h}\right)\right)\right\|_{0, \Gamma_{1, C}}^{2}+\left(\theta+\frac{\beta_{1}}{2}\right)\left\|\gamma^{\frac{1}{2}} R_{\hat{\rho}}\left(v^{h}-u^{h}\right)\right\|_{0, \Gamma_{1, C}}^{2} .
\end{aligned}
$$

For all $a, b \in \mathbb{R}$, we have the following estimate:

$$
\left([a]_{+}-[b]_{+}\right)(b-a) \leqslant-\left([a]_{+}-[b]_{+}\right)^{2} .
$$


Then, set:

$$
\begin{aligned}
\tau_{1} & =\int_{\Gamma_{1, C}} \frac{1}{\gamma}\left(\left[P_{\gamma}^{h, \hat{\rho}}\left(u^{h}\right)-g\right]_{+}-\left[P_{\gamma}^{h}(u)-g\right]_{+}\right) P_{\theta \gamma}^{h, \hat{\rho}}\left(v^{h}-u^{h}\right) \mathrm{d} \Gamma \\
& =\int_{\Gamma_{1, C}}\left(\sigma_{n}(u)+\frac{1}{\gamma}\left[P_{\gamma}^{h, \hat{\rho}}\left(u^{h}\right)-g\right]_{+}\right) P_{\theta \gamma}^{h, \hat{\rho}}\left(v^{h}-u^{h}\right) \mathrm{d} \Gamma .
\end{aligned}
$$

Using Cauchy-Schwarz and Young's inequalities for $\beta_{2}>0$ and $\beta_{3}>0$ and (20), it holds:

$$
\begin{aligned}
\tau_{1} \leqslant & \left(-1+\frac{1}{2 \beta_{2}}+\frac{|1-\theta|}{2 \beta_{3}}\right)\left\|\gamma^{\frac{1}{2}}\left(\sigma_{n}(u)+\frac{1}{\gamma}\left[P_{\gamma}^{h, \hat{\rho}}\left(u^{h}\right)-g\right]_{+}\right)\right\|_{0, \Gamma_{1, C}}^{2} \\
& +\frac{\beta_{2}}{2}\left\|\gamma^{-\frac{1}{2}}\left(P_{\gamma}^{h, \hat{\rho}}\left(v^{h}\right)-P_{\gamma}^{h}(u)\right)\right\|_{0, \Gamma_{1, C}}^{2}+\frac{|1-\theta| \beta_{3}}{2}\left\|\gamma^{\frac{1}{2}} R_{\hat{\rho}}\left(v^{h}-u^{h}\right)\right\|_{0, \Gamma_{1, C}}^{2} .
\end{aligned}
$$

Moreover, set:

$\tau_{2}$

$$
\begin{aligned}
= & -\sum_{i=1,2}\left\|\gamma^{-\frac{1}{2}}\left(u_{i}-u_{i}^{h}\right)\right\|_{0, \Gamma_{i, D}}^{2}-\sum_{i=1,2} \int_{\Gamma_{i, D}} \gamma^{-1}\left(v_{i}^{h}-u_{i}\right) \cdot\left(\bar{P}_{i, \gamma}^{h}\left(u_{i}\right)-\bar{P}_{i, \gamma}^{h, \rho}\left(u_{i}^{h}\right)\right) \mathrm{d} \Gamma \\
& +\sum_{i=1,2} \int_{\Gamma_{i, D}}\left(u_{i}-u_{i}^{h}\right) \cdot\left(\sigma\left(u_{i}\right) n_{i}-\bar{R}_{\hat{\rho}}\left(v_{i}^{h}\right)\right) \mathrm{d} \Gamma \\
& +(1+\theta) \sum_{i=1,2} \int_{\Gamma_{i, D}}\left(u_{i}-u_{i}^{h}\right) \cdot\left(\bar{R}_{\hat{\rho}}\left(v_{i}^{h}-u_{i}^{h}\right)\right) \mathrm{d} \Gamma
\end{aligned}
$$

Using Young's inequality for $\beta_{4}>0$, it holds:

$$
\begin{aligned}
& -\sum_{i=1,2} \int_{\Gamma_{i, D}} \gamma^{-1}\left(v_{i}^{h}-u_{i}\right) \cdot\left(\bar{P}_{i, \gamma}^{h}\left(u_{i}\right)-\bar{P}_{i, \gamma}^{h, \rho}\left(u_{i}^{h}\right)\right) \mathrm{d} \Gamma \\
\leqslant & \frac{\beta_{4}}{2} \sum_{i=1,2}\left\|\gamma^{-\frac{1}{2}}\left(v_{i}^{h}-u_{i}\right)\right\|_{0, \Gamma_{i, D}}^{2}+\frac{1}{2 \beta_{4}} \sum_{i=1,2}\left\|\gamma^{-\frac{1}{2}}\left(\bar{P}_{i, \gamma}^{h}\left(u_{i}\right)-\bar{P}_{i, \gamma}^{h, \rho}\left(u_{i}^{h}\right)\right)\right\|_{0, \Gamma_{i, D}}^{2} \\
\leqslant & \frac{\beta_{4}}{2} \sum_{i=1,2}\left\|\gamma^{-\frac{1}{2}}\left(v_{i}^{h}-u_{i}\right)\right\|_{0, \Gamma_{i, D}}^{2}+\frac{1}{\beta_{4}} \sum_{i=1,2}\left\|\gamma^{-\frac{1}{2}}\left(u_{i}-u_{i}^{h}\right)\right\|_{0, \Gamma_{i, D}}^{2} \\
& +\frac{1}{\beta_{4}} \sum_{i=1,2}\left\|\gamma^{\frac{1}{2}}\left(\sigma\left(u_{i}\right) n_{i}-\bar{R}_{\hat{\rho}}\left(u_{i}^{h}\right)\right)\right\|_{0, \Gamma_{i, D}}^{2}
\end{aligned}
$$

and for $\beta_{5}>0$

$$
\begin{aligned}
& \sum_{i=1,2} \int_{\Gamma_{i, D}}\left(u_{i}-u_{i}^{h}\right) \cdot\left(\sigma\left(u_{i}\right) n_{i}-\bar{R}_{\hat{\rho}}\left(v_{i}^{h}\right)\right) \mathrm{d} \Gamma \\
\leqslant & \frac{1}{2 \beta_{5}} \sum_{i=1,2}\left\|\gamma^{-\frac{1}{2}}\left(u_{i}-u_{i}^{h}\right)\right\|_{0, \Gamma_{i, D}}^{2}+\frac{\beta_{5}}{2} \sum_{i=1,2}\left\|\gamma^{\frac{1}{2}}\left(\sigma\left(u_{i}\right) n_{i}-\bar{R}_{\hat{\rho}}\left(v_{i}^{h}\right)\right)\right\|_{0, \Gamma_{i, D}}^{2},
\end{aligned}
$$

and for $\beta_{6}>0$

$$
\begin{aligned}
& (1+\theta) \sum_{i=1,2} \int_{\Gamma_{i, D}}\left(u_{i}-u_{i}^{h}\right) \cdot\left(\bar{R}_{\hat{\rho}}\left(v_{i}^{h}-u_{i}^{h}\right)\right) \mathrm{d} \Gamma \\
\leqslant & \frac{|1+\theta|}{2 \beta_{6}} \sum_{i=1,2}\left\|\gamma^{-\frac{1}{2}}\left(u_{i}-u_{i}^{h}\right)\right\|_{0, \Gamma_{i, D}}^{2}+\frac{\beta_{6}|1+\theta|}{2} \sum_{i=1,2}\left\|\gamma^{\frac{1}{2}}\left(\bar{R}_{\hat{\rho}}\left(v_{i}^{h}-u_{i}^{h}\right)\right)\right\|_{0, \Gamma_{i, D}}^{2} .
\end{aligned}
$$


Using inequalities (23), (24) and (25) in $\tau_{2}+\frac{1}{2} \sum_{i=1,2}\left\|\gamma^{-\frac{1}{2}}\left(u_{i}-u_{i}^{h}\right)\right\|_{0, \Gamma_{i, D}}^{2}$, it holds:

$$
\begin{aligned}
& \tau_{2}+\frac{1}{2} \sum_{i=1,2}\left\|\gamma^{-\frac{1}{2}}\left(u_{i}-u_{i}^{h}\right)\right\|_{0, \Gamma_{i, D}}^{2} \\
\leqslant & \left(-\frac{1}{2}+\frac{1}{\beta_{4}}+\frac{1}{2 \beta_{5}}+\frac{|1+\theta|}{2 \beta_{6}}\right) \sum_{i=1,2}\left\|\gamma^{-\frac{1}{2}}\left(u_{i}-u_{i}^{h}\right)\right\|_{0, \Gamma_{i, D}}^{2} \\
& +\frac{\beta_{4}}{2} \sum_{i=1,2}\left\|\gamma^{-\frac{1}{2}}\left(v_{i}^{h}-u_{i}\right)\right\|_{0, \Gamma_{i, D}}^{2}+\frac{1}{\beta_{4}} \sum_{i=1,2}\left\|\gamma^{\frac{1}{2}}\left(\sigma\left(u_{i}\right) n_{i}-\bar{R}_{\hat{\rho}}\left(u_{i}^{h}\right)\right)\right\|_{0, \Gamma_{i, D}}^{2} \\
& +\frac{\beta_{5}}{2} \sum_{i=1,2}\left\|\gamma^{\frac{1}{2}}\left(\sigma\left(u_{i}\right) n_{i}-\bar{R}_{\hat{\rho}}\left(v_{i}^{h}\right)\right)\right\|_{0, \Gamma_{i, D}}^{2}+\frac{\beta_{6}|1+\theta|}{2} \sum_{i=1,2}\left\|\gamma^{\frac{1}{2}}\left(\bar{R}_{\hat{\rho}}\left(v_{i}^{h}-u_{i}^{h}\right)\right)\right\|_{0, \Gamma_{i, D}}^{2} .
\end{aligned}
$$

Gathering (18), (19), (21) and (26) in (17), it holds:

$$
\begin{aligned}
\frac{\alpha}{2} \sum_{i=1,2}\left\|u_{i}-u_{i}^{h}\right\|_{1, \Omega_{i}}^{2} \leqslant & \frac{C^{2}}{2 \alpha} \sum_{i=1,2}\left\|u_{i}-v_{i}^{h}\right\|_{1, \Omega_{i}}^{2} \\
& +\frac{\theta^{2}}{2 \beta_{1}}\left\|\gamma^{\frac{1}{2}}\left(\sigma_{n}(u)-R_{\hat{\rho}}\left(v^{h}\right)\right)\right\|_{0, \Gamma_{1, C}}^{2} \\
& +\left(\theta+\frac{\beta_{1}}{2} \frac{|1-\theta| \beta_{3}}{2}\right)\left\|\gamma^{\frac{1}{2}} R_{\hat{\rho}}\left(v^{h}-u^{h}\right)\right\|_{0, \Gamma_{1, C}}^{2} \\
& +\left(-1+\frac{1}{2 \beta_{2}}+\frac{|1-\theta|}{2 \beta_{3}}\right)\left\|\gamma^{\frac{1}{2}}\left(\sigma_{n}(u)+\frac{1}{\gamma}\left[P_{\gamma}^{h, \rho}\left(u^{h}\right)-g\right]_{+}\right)\right\|_{0, \Gamma_{1, C}}^{2} \\
& +\frac{\beta_{2}}{2}\left\|\gamma^{-\frac{1}{2}}\left(P_{\gamma}^{h, \rho}\left(v^{h}\right)-P_{\gamma}^{h}(u)\right)\right\|_{0, \Gamma_{1, C}}^{2} \\
& +\left(-\frac{1}{2}+\frac{1}{\beta_{4}}+\frac{1}{2 \beta_{5}}+\frac{|1+\theta|}{2 \beta_{6}}\right) \sum_{i=1,2}\left\|\gamma^{-\frac{1}{2}}\left(u_{i}-u_{i}^{h}\right)\right\|_{0, \Gamma_{i, D}}^{2} \\
& +\frac{\beta_{4}}{2} \sum_{i=1,2}\left\|\gamma^{-\frac{1}{2}}\left(v_{i}^{h}-u_{i}\right)\right\|_{0, \Gamma_{i, D}}^{2} \\
& +\frac{1}{\beta_{4}} \sum_{i=1,2}\left\|\gamma^{\frac{1}{2}}\left(\sigma\left(u_{i}\right) n_{i}-\bar{R}_{\hat{\rho}}\left(u_{i}^{h}\right)\right)\right\|_{0, \Gamma_{i, D}}^{2} \\
& +\frac{\beta_{5}}{2} \sum_{i=1,2}\left\|\gamma^{\frac{1}{2}}\left(\sigma\left(u_{i}\right) n_{i}-\bar{R}_{\hat{\rho}}\left(v_{i}^{h}\right)\right)\right\|_{0, \Gamma_{i, D}}^{2} \\
& +\frac{\beta_{6}|1+\theta|}{2} \sum_{i=1,2}\left\|\gamma^{\frac{1}{2}}\left(\bar{R}_{\hat{\rho}}\left(v_{i}^{h}-u_{i}^{h}\right)\right)\right\|_{0, \Gamma_{i, D}}^{2}
\end{aligned}
$$

Using lemmas 4.2 and 4.5 , we obtain:

$$
\begin{aligned}
\left\|\gamma^{\frac{1}{2}} R_{\hat{\rho}}\left(v^{h}-u^{h}\right)\right\|_{0, \Gamma_{1, C}}^{2} & \leqslant C_{1} \gamma_{0} \sum_{i=1,2}\left\|v_{i}^{h}-u_{i}^{h}\right\|_{1, \Omega_{i}}^{2} \\
& \leqslant 2 C_{1} \gamma_{0}\left(\sum_{i=1,2}\left\|v_{i}^{h}-u_{i}\right\|_{1, \Omega_{i}}^{2}+\sum_{i=1,2}\left\|u_{i}-u_{i}^{h}\right\|_{1, \Omega_{i}}^{2}\right), \\
\sum_{i=1,2}\left\|\gamma^{\frac{1}{2}} R_{\hat{\rho}}\left(v_{i}^{h}-u_{i}^{h}\right)\right\|_{0, \Gamma_{i, D}}^{2} & \leqslant C_{2} \gamma_{0} \sum_{i=1,2}\left\|v_{i}^{h}-u_{i}^{h}\right\|_{1, \Omega_{i}}^{2} \\
& \leqslant 2 C_{2} \gamma_{0}\left(\sum_{i=1,2}\left\|v_{i}^{h}-u_{i}\right\|_{1, \Omega_{i}}^{2}+\sum_{i=1,2}\left\|u_{i}-u_{i}^{h}\right\|_{1, \Omega_{i}}^{2}\right),
\end{aligned}
$$


and we know

$$
\left\|\gamma^{-\frac{1}{2}} P_{\gamma}^{h, \rho}\left(v^{h}\right)-P_{\gamma}^{h}(u)\right\|_{0, \Gamma_{1, C}}^{2} \leqslant 2\left\|\gamma^{-\frac{1}{2}}\left(v^{h}-u\right)\right\|_{0, \Gamma_{1, C}}^{2}+2\left\|\gamma^{\frac{1}{2}}\left(R_{\hat{\rho}}\left(v^{h}\right)-\sigma_{n}(u)\right)\right\|_{0, \Gamma_{1, C}}^{2},
$$

and using Young's inequality for $\beta>0$, it holds:

$$
\begin{aligned}
-\sum_{i=1,2}\left\|\gamma^{-\frac{1}{2}}\left(u_{i}-u_{i}^{h}\right)\right\|_{0, \Gamma_{i, D}}^{2} \leqslant & -\left(1-\frac{1}{\beta}\right) \sum_{i=1,2}\left\|\gamma^{\frac{1}{2}} \sigma\left(u_{i}\right) n_{i}-R_{\hat{\rho}}\left(u_{i}^{h}\right)\right\|_{0, \Gamma_{i, D}}^{2} \\
& -(1-\beta) \sum_{i=1,2}\left\|\gamma^{-\frac{1}{2}} \bar{P}_{\gamma}^{h}(u)-\bar{P}_{\gamma}^{h, \rho}\left(u^{h}\right)\right\|_{0, \Gamma_{i, D}}^{2}
\end{aligned}
$$

Let $\theta \in \mathbb{R}$ be fixed, if $\beta_{2}, \beta_{3}, \beta_{4}, \beta_{5}$ and $\beta_{6}$ are chosen sufficiently large such that:

$$
\begin{gathered}
-1+\frac{1}{2 \beta_{2}}+\frac{|1-\theta|}{2 \beta_{3}}<-\frac{1}{2}, \\
-\frac{1}{2}+\frac{1}{\beta_{4}}+\frac{1}{2 \beta_{5}}+\frac{|1+\theta|}{2 \beta_{6}}<-\frac{1}{4} .
\end{gathered}
$$

And if $\gamma_{0}$ is sufficiently small and $\beta<1$, we get the inequality (16).

In the case $\theta=-1$, thanks to (27), it holds:

$$
\begin{aligned}
\frac{\alpha}{2} \sum_{i=1,2}\left\|u_{i}-u_{i}^{h}\right\|_{1, \Omega_{i}}^{2} \leqslant & \frac{C^{2}}{2 \alpha} \sum_{i=1,2}\left\|u_{i}-v_{i}^{h}\right\|_{1, \Omega_{i}}^{2} \\
& +\frac{1}{2 \beta_{1}}\left\|\gamma^{\frac{1}{2}}\left(\sigma_{n}(u)-R_{\hat{\rho}}\left(v^{h}\right)\right)\right\|_{0, \Gamma_{1, C}}^{2} \\
& +\left(-1+\frac{\beta_{1}}{2}+\beta_{3}\right)\left\|\gamma^{\frac{1}{2}} R_{\hat{\rho}}\left(v^{h}-u^{h}\right)\right\|_{0, \Gamma_{1, C}}^{2} \\
& +\left(-1+\frac{1}{2 \beta_{2}}+\frac{1}{\beta_{3}}\right)\left\|\gamma^{\frac{1}{2}}\left(\sigma_{n}(u)+\frac{1}{\gamma}\left[P_{\gamma}\left(u^{h}\right)-g\right]_{+}\right)\right\|_{0, \Gamma_{1, C}}^{2} \\
& +\frac{\beta_{2}}{2}\left\|\gamma^{-\frac{1}{2}} P_{\gamma}^{h, \rho}\left(v^{h}\right)-P_{\gamma}^{h}(u)\right\|_{0, \Gamma_{1, C}}^{2} \\
& +\left(-\frac{1}{2}+\frac{1}{\beta_{4}}+\frac{1}{2 \beta_{5}}\right) \sum_{i=1,2}\left\|\gamma^{-\frac{1}{2}}\left(u_{i}-u_{i}^{h}\right)\right\|_{0, \Gamma_{i, D}}^{2} \\
& +\frac{\beta_{4}}{2} \sum_{i=1,2}\left\|\gamma^{-\frac{1}{2}}\left(v_{i}^{h}-u_{i}\right)\right\|_{0, \Gamma_{i, D}}^{2}+\frac{1}{\beta_{4}} \sum_{i=1,2}\left\|\gamma^{\frac{1}{2}}\left(\sigma\left(u_{i}\right) n_{i}-\bar{R}_{\hat{\rho}}\left(u_{i}^{h}\right)\right)\right\|_{0, \Gamma_{i, D}}^{2} \\
& +\frac{\beta_{5}}{2} \sum_{i=1,2}\left\|\gamma^{\frac{1}{2}}\left(\sigma\left(u_{i}\right) n_{i}-\bar{R}_{\hat{\rho}}\left(v_{i}^{h}\right)\right)\right\|_{0, \Gamma_{i, D}}^{2} \cdot
\end{aligned}
$$

Let $\eta_{1}>0$ and $\eta_{2}>0$, we take $\beta_{1}=2 \eta_{1}, \beta_{2}=1+1 / \eta_{1}, \beta_{3}=1+\eta_{1}, \beta_{4}=2\left(1+\eta_{2}\right)$, 
$\beta_{5}=2\left(1+1 / \eta_{2}\right)$, then it holds:

$$
\begin{aligned}
\frac{\alpha}{2} \sum_{i=1,2}\left\|u_{i}-u_{i}^{h}\right\|_{1, \Omega_{i}}^{2} \leqslant & \frac{C^{2}}{2 \alpha} \sum_{i=1,2}\left\|u_{i}-v_{i}^{h}\right\|_{1, \Omega_{i}}^{2} \\
& +\frac{1}{4 \eta_{1}}\left\|\gamma^{\frac{1}{2}}\left(\sigma_{n}(u)-R_{\hat{\rho}}\left(v^{h}\right)\right)\right\|_{0, \Gamma_{1, C}}^{2}+2 \eta_{1}\left\|\gamma^{\frac{1}{2}} R_{\hat{\rho}}\left(v^{h}-u^{h}\right)\right\|_{0, \Gamma_{1, C}}^{2} \\
& -\frac{\eta_{1}}{2\left(1+\eta_{1}\right)}\left\|\gamma^{\frac{1}{2}}\left(\sigma_{n}(u)+\frac{1}{\gamma}\left[P_{\gamma}\left(u^{h}\right)-g\right]+\right)\right\|_{0, \Gamma_{1, C}}^{2} \\
& +\frac{1+\eta_{1}}{2 \eta_{1}}\left\|\gamma^{-\frac{1}{2}} P_{\gamma}^{h, \rho}\left(v^{h}\right)-P_{\gamma}^{h}(u)\right\|_{0, \Gamma_{1, C}}^{2} \\
& -\frac{\eta_{2}}{4\left(1+\eta_{2}\right)} \sum_{i=1,2}\left\|\gamma^{-\frac{1}{2}}\left(u_{i}-u_{i}^{h}\right)\right\|_{0, \Gamma_{i, D}}^{2} \\
& +\left(1+\eta_{2}\right) \sum_{i=1,2}\left\|\gamma^{-\frac{1}{2}}\left(v_{i}^{h}-u_{i}\right)\right\|_{0, \Gamma_{i, D}}^{2} \\
& +\frac{1}{2\left(1+\eta_{2}\right)} \sum_{i=1,2}\left\|\gamma^{\frac{1}{2}}\left(\sigma\left(u_{i}\right) n_{i}-\bar{R}_{\hat{\rho}}\left(u_{i}^{h}\right)\right)\right\|_{0, \Gamma_{i, D}}^{2} \\
& +\left(1+1 / \eta_{2}\right) \sum_{i=1,2}\left\|\gamma^{\frac{1}{2}}\left(\sigma\left(u_{i}\right) n_{i}-\bar{R}_{\hat{\rho}}\left(v_{i}^{h}\right)\right)\right\|_{0, \Gamma_{i, D}}^{2}
\end{aligned}
$$

Using (28) and $\beta=\frac{\eta_{2}}{2+\eta_{2}}<1$, we have:

$$
\begin{aligned}
-\frac{\eta_{2}}{4\left(1+\eta_{2}\right)} \sum_{i=1,2}\left\|\gamma^{-\frac{1}{2}}\left(u_{i}-u_{i}^{h}\right)\right\|_{0, \Gamma_{i, D}}^{2} \leqslant & \frac{1}{2\left(1+\eta_{2}\right)} \sum_{i=1,2}\left\|\gamma^{\frac{1}{2}} \sigma\left(u_{i}\right) n_{i}-R_{\hat{\rho}}\left(u_{i}^{h}\right)\right\|_{0, \Gamma_{i, D}}^{2} \\
& -\frac{\eta_{2}}{2\left(1+\eta_{2}\right)\left(2+\eta_{2}\right)} \sum_{i=1,2}\left\|\gamma^{-\frac{1}{2}} \bar{P}_{\gamma}^{h}(u)-\bar{P}_{\gamma}^{h, \rho}\left(u^{h}\right)\right\|_{0, \Gamma_{i, D}}^{2},
\end{aligned}
$$

and

$$
\begin{aligned}
\sum_{i=1,2}\left\|\gamma^{\frac{1}{2}} \sigma\left(u_{i}\right) n_{i}-R_{\hat{\rho}}\left(u_{i}^{h}\right)\right\|_{0, \Gamma_{i, D}}^{2} \leqslant & 2 \sum_{i=1,2}\left\|\gamma^{\frac{1}{2}} \sigma\left(u_{i}\right) n_{i}-R_{\hat{\rho}}\left(v_{i}^{h}\right)\right\|_{0, \Gamma_{i, D}}^{2} \\
& +2 \sum_{i=1,2} \| \gamma^{\frac{1}{2}} \sigma\left(R_{\hat{\rho}}\left(v_{i}^{h}-u_{i}^{h}\right) \|_{0, \Gamma_{i, D}}^{2} .\right.
\end{aligned}
$$

Let $\gamma_{0}$ be positive. If we take $\eta_{1}=\alpha /\left(32 C_{1} \gamma_{0}\right)$ and $\eta_{2}=C_{2} \gamma_{0} /(32 \alpha)$, then we get the inequality (16). This ends the proof of Theorem 4.8

Theorem 4.9. Let $u$ be a solution of the variational problem (4). Suppose that $u$ belongs to $\left(H^{\frac{3}{2}+\nu}\left(\Omega_{1}\right)\right)^{d} \times\left(H^{\frac{3}{2}+\nu}\left(\Omega_{2}\right)\right)^{d}$ with $1 / 2 \geqslant \nu>0$ if $k=1$ and with $1>\nu>0$ if $k=2$. Then, if additionally $\gamma_{0}>0$ is sufficiently small when $\theta \neq-1$, the solution $u^{h}$ of the stabilize problem (10) satisfies the following a priori error estimate:

$$
\begin{aligned}
& \sum_{i=1,2}\left\|u_{i}-u_{i}^{h}\right\|_{1, \Omega_{i}}^{2}+\left\|\gamma^{\frac{1}{2}}\left(\sigma_{n}(u)+\frac{1}{\gamma}\left[P_{\gamma}^{h, \hat{\rho}}\left(u^{h}\right)-g\right]_{+}\right)\right\|_{0, \Gamma_{1, C}}^{2} \\
& +\sum_{i=1,2}\left\|\gamma^{-\frac{1}{2}}\left(\bar{P}_{i, \gamma}^{h, \hat{\rho}}\left(u_{i}^{h}\right)-\bar{P}_{i, \gamma}^{h}\left(u_{i}\right)\right)\right\|_{0, \Gamma_{i, D}}^{2} \leqslant C h^{1+2 \nu} \sum_{i=1,2}\|u\|_{\frac{3}{2}+\nu, \Omega_{i}}^{2}
\end{aligned}
$$

with $C>0$ a constant independent of $h$ and $u$. 
Proof. Now let us establish the inequality (30). Set $v_{i}^{h}=\Pi_{i}^{h}\left(u_{i}\right)$, we have the following estimates:

$$
\begin{gathered}
\left\|u_{i}-\Pi_{i}^{h}\left(u_{i}\right)\right\|_{m, \Omega_{i}} \leqslant C h^{k+1-m}\left\|u_{i}\right\|_{k+1, \Omega_{i}}, \\
\left\|R_{\hat{\rho}}\left(\Pi^{h}(u)\right)-\sigma_{n}(u)\right\|_{0, \Gamma_{1, C}}^{2} \leqslant C h^{2 k-1} \sum_{i=1,2}\|u\|_{k+1, \Omega_{i}}^{2},
\end{gathered}
$$

and

$$
\sum_{i=1,2}\left\|\bar{R}_{\hat{\rho}}\left(\Pi_{i}^{h}\left(u_{i}\right)\right)-\sigma\left(u_{i}\right) n_{i}\right\|_{0, \Gamma_{i, D}}^{2} \leqslant C h^{2 k-1} \sum_{i=1,2}\|u\|_{k+1, \Omega_{i}}^{2} .
$$

If we replace $v_{i}^{h}$ by $\Pi_{i}^{h}\left(u_{i}\right)$ in (16), $\gamma=\gamma_{0} h$ and we use the previous inequalities, we get (30). We can write:

$$
\begin{aligned}
& \sum_{i=1,2}\left\|u_{i}-u_{i}^{h}\right\|_{1, \Omega_{i}}+\left\|\gamma^{\frac{1}{2}}\left(\sigma_{n}(u)+\frac{1}{\gamma}\left[P_{\gamma}^{h, \hat{\rho}}\left(u^{h}\right)-g\right]_{+}\right)\right\|_{0, \Gamma_{1, C}} \\
& +\sum_{i=1,2}\left\|\gamma^{-\frac{1}{2}}\left(\bar{P}_{i, \gamma}^{h, \hat{\rho}}\left(u_{i}^{h}\right)-\bar{P}_{i, \gamma}^{h}\left(u_{i}\right)\right)\right\|_{0, \Gamma_{i, D}} \leqslant C h^{1 / 2+\nu} \sum_{i=1,2}\|u\|_{\frac{3}{2}+\nu, \Omega_{i}}
\end{aligned}
$$

\section{$5 \quad$ Numerical study}

This section is dedicated to some numerical experiments with isoparametric Lagrange $P 1$ or $P 2$ finite element methods. The accuracy of the method is discussed for the different cases with respect to the finite element used, the mesh size and the value of the parameter $\gamma_{0}$. Note that the following results are obtained without the stabilization introduced in Section 3.4. From a numerical viewpoint, the stabilization seems not strictly necessary to obtain an optimal rate of convergence. This has already been observed in a linear case in [16]. The numerical tests in two dimensions (resp. three dimensions) are performed on a fictitious domain $\Omega=]-0.5,0.5\left[^{2}\right.$ (resp. $\Omega=]-0.5,0.5\left[{ }^{3}\right.$ which contains the first body $\Omega_{1}$, a circle of radius 0.25 and center $(0,0)$ (resp. a sphere of radius 0.25 and center $(0,0,0)$ ), and the second $\left.\Omega_{2}=\right]-0.5,0.5[\times]-0.5,-0.25[$ (resp. $\left.\Omega_{2}=\right]-0.5,0.5\left[^{2} \times\right]-0.5,-0.25[$ ). A Dirichlet condition is prescribed on the bottom of the rectangle (resp. parallelepiped).

The projector $\Pi$ is defined from the lower part of the boundary of $\Omega_{1}$ (i.e. for $\Gamma_{1, C}=\{x \in$ $\left.\left.\partial \Omega_{1}: x_{d} \leq 0\right\}\right)$ onto its projection on the top boundary of $\Omega_{2}$. All remaining parts of the boundaries of $\Omega_{1}$ and $\Omega_{2}$ are considered traction free.

Since no Dirichlet condition is applied on $\Omega_{1}$, the problem is only semi-coercive. In order to recover the uniqueness of the solution, it is needed to prescribe the horizontal rigid translation in $2 \mathrm{D}$ and two horizontal translations and one rotation in $3 \mathrm{D}$. This is done by prescribing the displacement on some given convenient points.

We use a generalized Newton's method to solve the discrete problem (10) (see [27] for more details) and our finite element library GetFEM $++^{1}$. The tool for fictitious domain methods of GetFEM++ has been used which provides cut integration methods. The geometries are described with zero level sets of some signed distances to the domain boundaries. The distance functions are approximated by quadratic Lagrange finite elements. In order to build cut integration methods,

\footnotetext{
${ }^{1}$ see http://download.gna.org/getfem/html/homepage/
} 
each element of the mesh which crosses a domain boundary is cut into a set of sub-elements conforming to this boundary. Then, an integration method is produced on each sub-element lying on the interior of a domain and on each sub-element boundary lying on a domain boundary. In order to obtain a convenient order for the produced integration methods and for the approximation of the domain boundaries, curved sub-elements are used.

Moreover, no specific treatment have been considered for the fact that boundary terms for the contact condition approximated by Nitsche's method is non-regular (due to the positive part). We used an order four numerical integration method on each sub-element and we noted no improvement of the accuracy with higher order or refined numerical integration method.

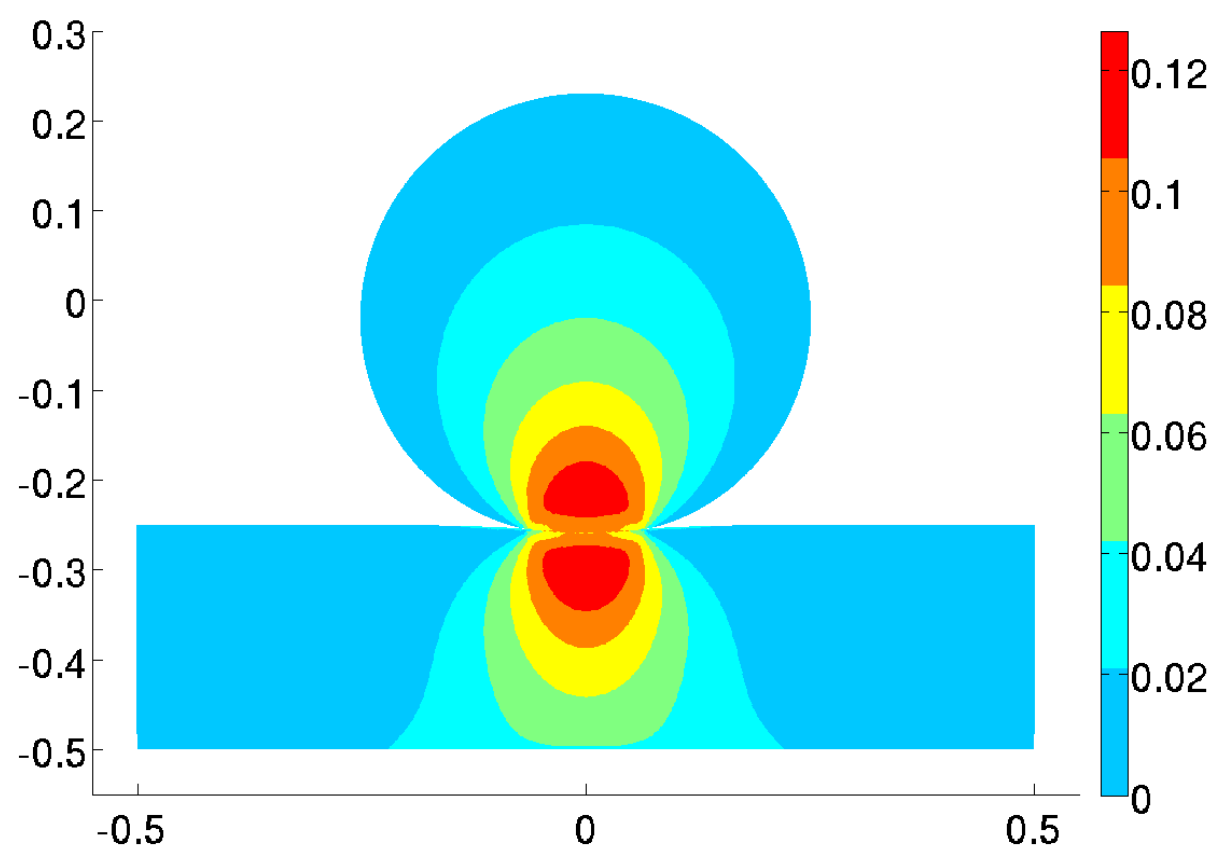

Figure 4: 2D numerical reference solution with contour plot of Von Mises stress. Parameters $h=1 / 400, \gamma_{0}=1 / 200, \theta=-1$ and $P_{2}$ elements.

For simplicity, we consider a dimensionless situation with Lamé coefficients $\lambda=1$ and $\mu=1$ and a vertical volume density of force -0.1 .

The situation studied is not strictly speaking of Hertz type due to the fact that $\Omega_{2}$ is bounded. The expression of the exact solution being unknown, the convergence is studied with respect to a reference solution computed with a $P_{2}$ isoparametric element on a very fine mesh $(h=1 / 200$ in $2 \mathrm{D}$ and $h=1 / 30$ in $3 \mathrm{D}$ ) with the skew-symmetric method $\theta=-1$ (see Figures 4 and 5 ).

\subsection{Numerical convergence in the two dimensional case}

We perform a numerical convergence study on the three methods $\theta=1, \theta=0$ and $\theta=-1$ for a fixed parameter $\gamma_{0}=1 / 200$ (chosen small in order to have the convergence for the three cases). On figures 6, 7 and 8, the relative error in percentage in $L^{2}$ and $H^{1}$-norms on each bodies for $P_{1}$ Lagrange finite elements are plotted. As expected the optimal convergence is obtained in $H^{1}$-norm for all methods in good accordance with Theorem 4.9. The rate of convergence in 


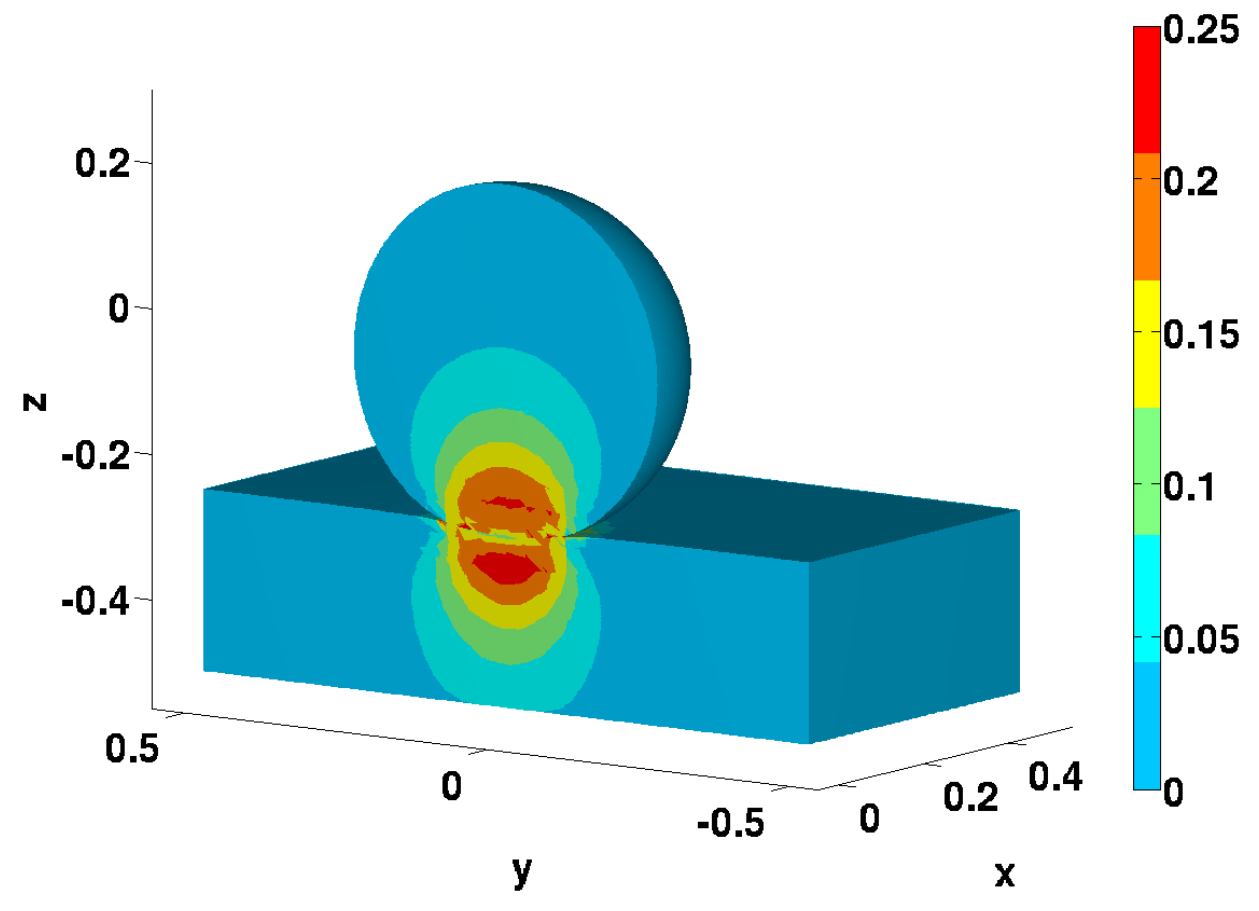

Figure 5: Cross-section of 3D numerical reference solution with contour plot of Von Mises stress. Parameters $h=1 / 30, \gamma_{0}=1 / 200, \theta=-1$ and $P_{2}$ elements.

$L^{2}$-norm is slightly sub-optimal on $\Omega_{2}$ if one refers to Aubin-Nitsche lemma in the linear case. However, such a result is not available for the nonlinear contact problem. Moreover, this slight sub-optimal convergence may be caused by the Neumann-Dirichlet transition at the bottom of $\Omega_{2}$.
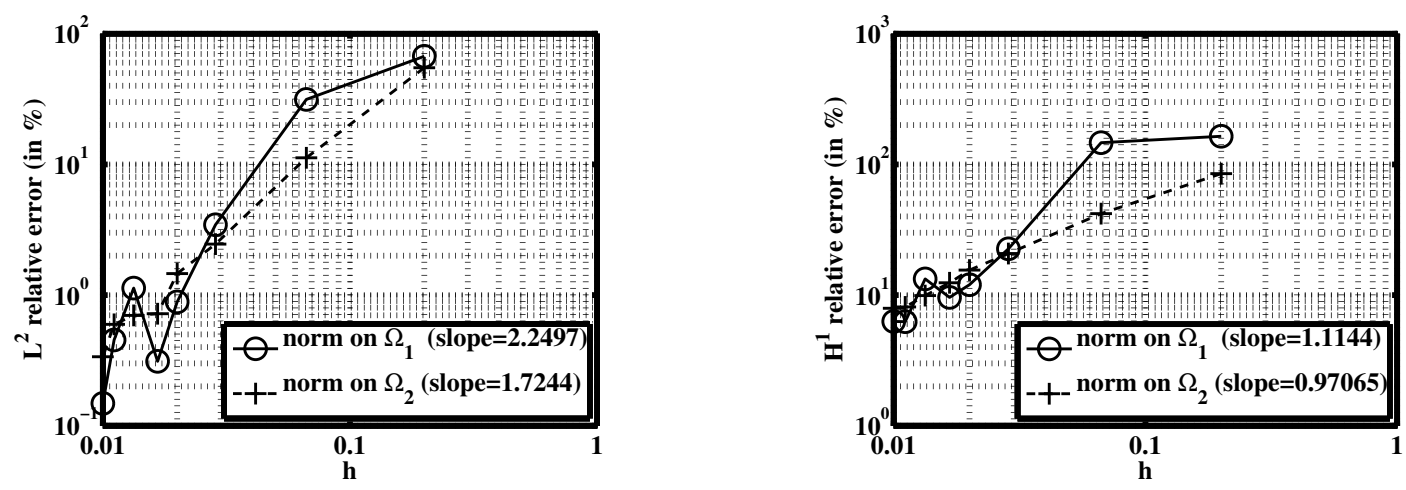

Figure 6: Convergence curves in 2D for the method $\theta=1$, with $\gamma_{0}=1 / 200$ and $P 1$ finite elements for the relative $L^{2}$-norm of the error (on the left) and the relative $H^{1}$-norm of the error (on the right).

On figures 9, 10 and 11, the same experiments are reported but for $P_{2}$ isoparametric Lagrange finite elements. The convergence rate for the three cases is close to 1.6 on $\Omega_{1}$ and 1.3 on $\Omega_{2}$. This is also close to optimality if one takes into account that the expected maximal regularity of the 

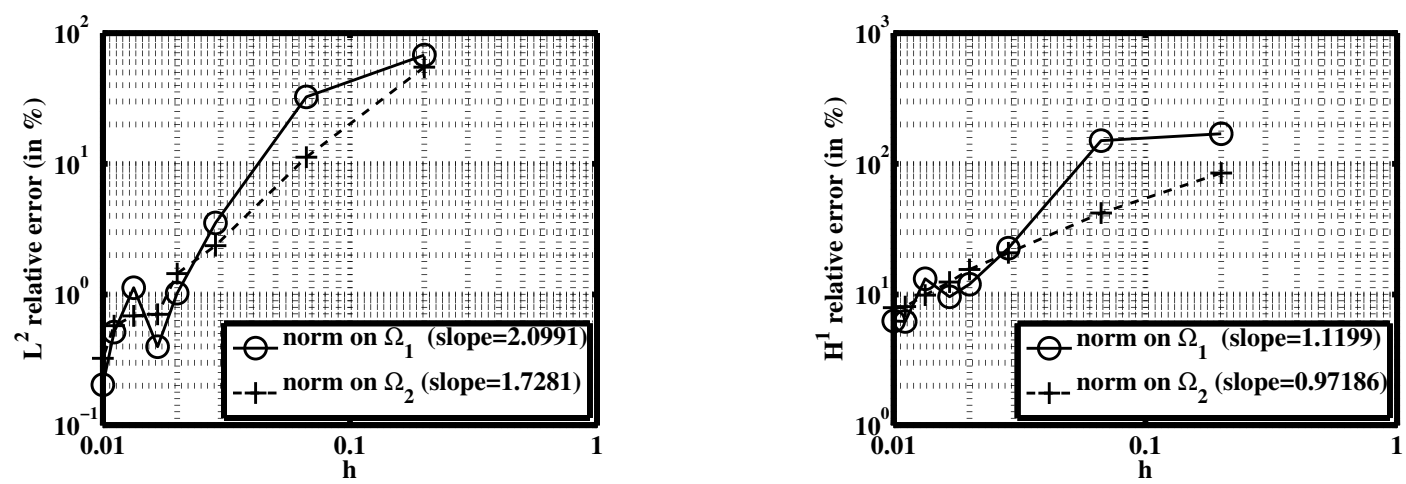

Figure 7: Convergence curves in 2D for the method $\theta=0$, with $\gamma_{0}=1 / 200$ and $P 1$ finite elements for the relative $L^{2}$-norm of the error (on the left) and the relative $H^{1}$-norm of the error (on the right).
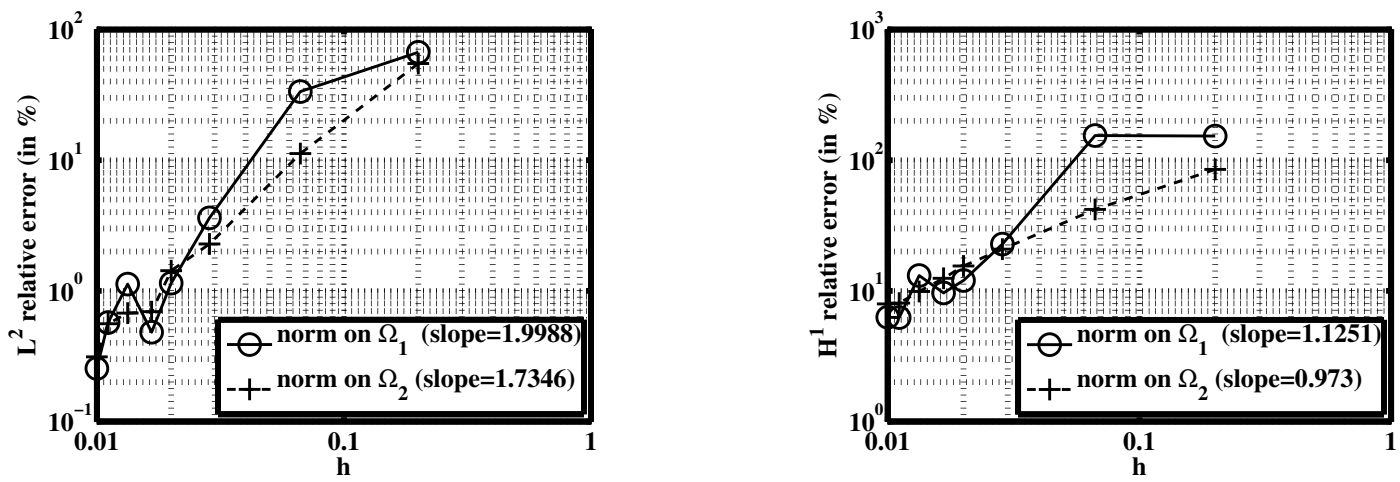

Figure 8: Convergence curves in 2D for the method $\theta=-1$, with $\gamma_{0}=1 / 200$ and $P 1$ finite elements for the relative $L^{2}$-norm of the error (on the left) and the relative $H^{1}$-norm of the error (on the right).

displacement next to the transition between contact and non-contact should be $H^{5 / 2-\eta}$ for any $\eta>0$ (However, this result has only been proved in a scalar case in [22]). Accordingly, one could expect that the convergence rate in the $L^{2}$-norm would be close to 2.5. This is approximately the case with again some sub-optimal rates which may due to the nonlinear characteristic of the contact condition or to the presence of non-regularities on the transition between the Dirichlet and the Neumann condition.

\subsection{Influence of the parameter $\gamma_{0}$}

The influence of $\gamma_{0}$ on the $H^{1}$-norm of the error is plotted in Figure 12 for $P_{1}$ elements and on Figure 13 for $P_{2}$ elements. The most affected method is the one for $\theta=1$. Indeed, it converges only for $\gamma_{0}$ very small. The large oscillation in the error norm comes from the fact that Newton's algorithm do not fully converge for all numerical experiments probably because there is no solution to the discrete problem in some cases. The method for $\theta=0$ gives a more regular error with respect to $\gamma_{0}$. It is still important to have $\gamma_{0}$ small to keep a good solution but a larger value is allowed. Accordingly to the theoretical result of Theorem 4.9, the influence of $\gamma_{0}$ on the method $\theta=-1$ is more limited. There is only a slight increase of the error for large values of $\gamma_{0}$. Note that 

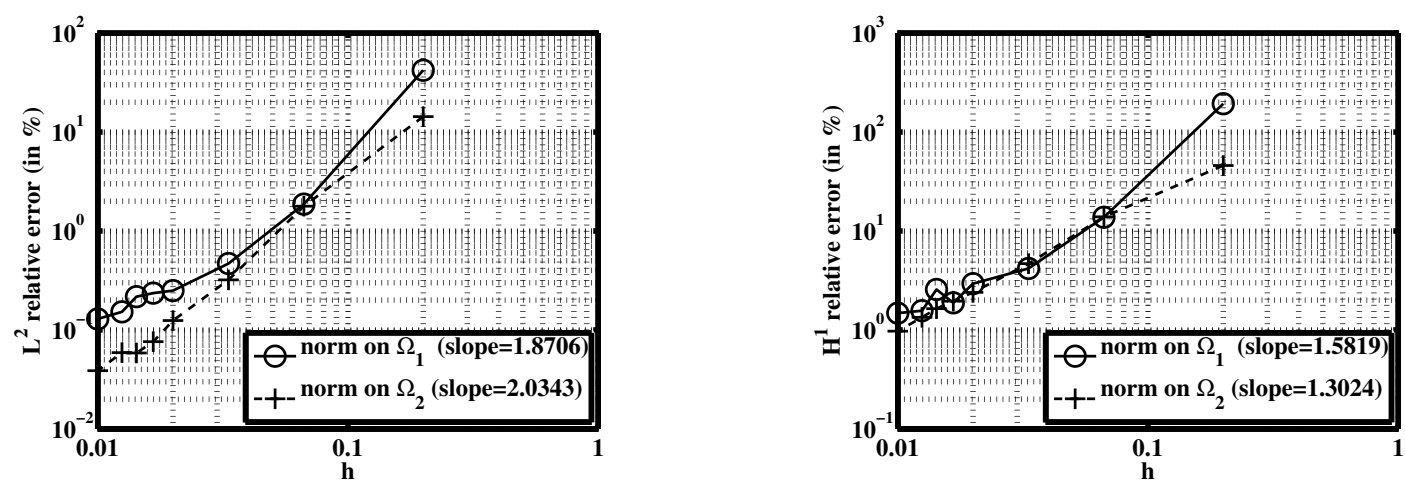

Figure 9: Convergence curves in 2D for the method $\theta=1$, with $\gamma_{0}=1 / 200$ and $P 2$ finite elements for the relative $L^{2}$-norm of the error (on the left) and the relative $H^{1}$-norm of the error (on the right).
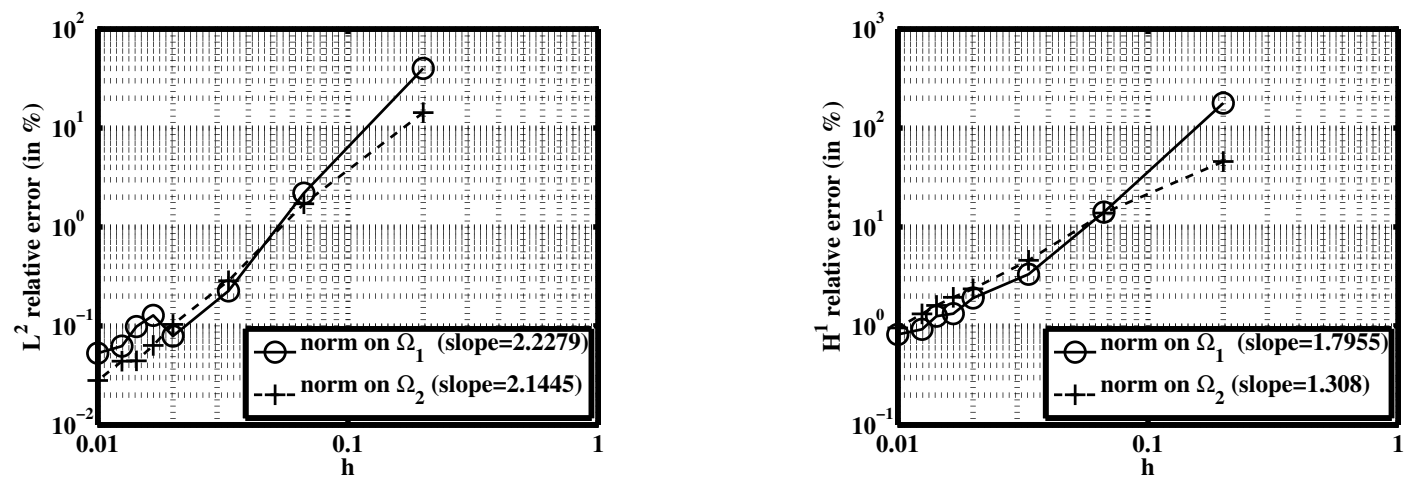

Figure 10: Convergence curves in 2D for the method $\theta=0$, with $\gamma_{0}=1 / 200$ and $P 2$ finite elements for the relative $L^{2}$-norm of the error (on the left) and the relative $H^{1}$-norm of the error (on the right).
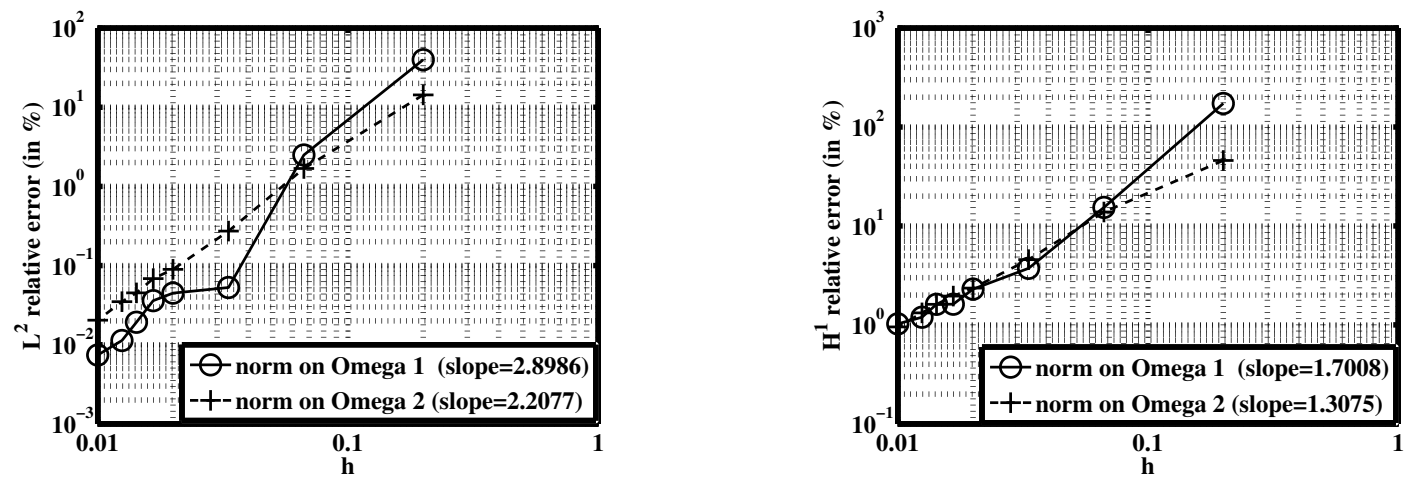

Figure 11: Convergence curves in 2D for the method $\theta=-1$, with $\gamma_{0}=1 / 200$ and $P 2$ finite elements for the relative $L^{2}$-norm of the error (on the left) and the relative $H^{1}$-norm of the error (on the right).

the nonlinear discrete system (10) becomes very stiff when $\gamma_{0}$ is very small. Thus, the possibility 

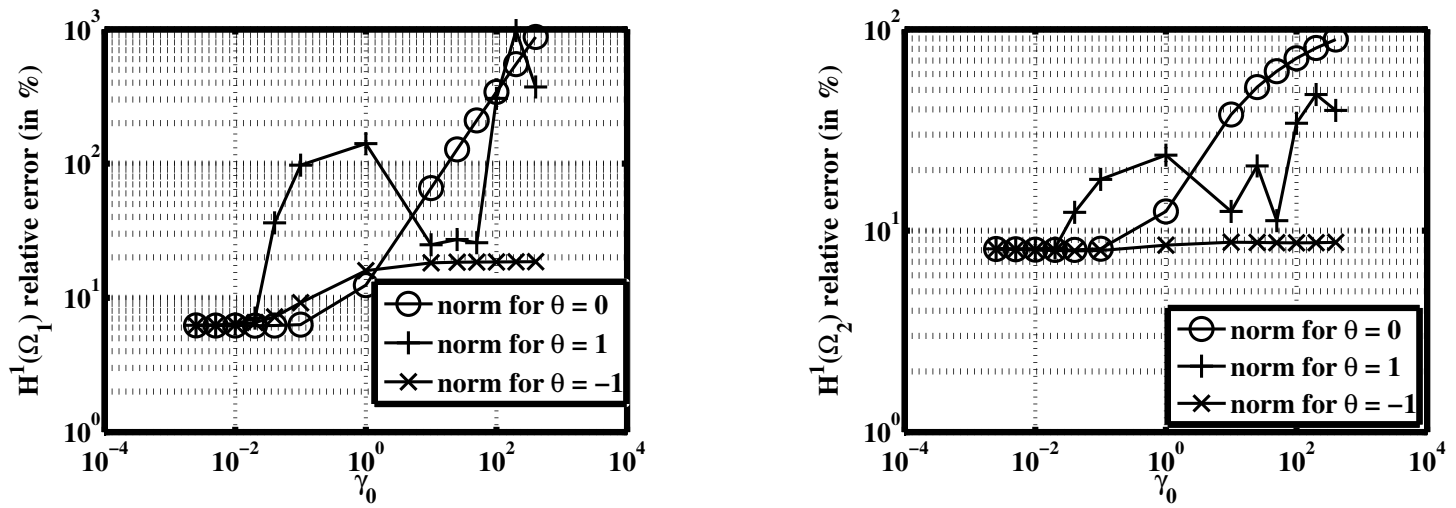

Figure 12: Influence of $\gamma_{0}$ on the relative $H^{1}$-norm of the error on $\Omega_{1}$ (on the left) and on $\Omega_{2}$ (on the right) in $2 \mathrm{D}$ for $\mathrm{h}=1 / 90$ and $P_{1}$ elements.

to have a large $\gamma_{0}$ is an advantage.
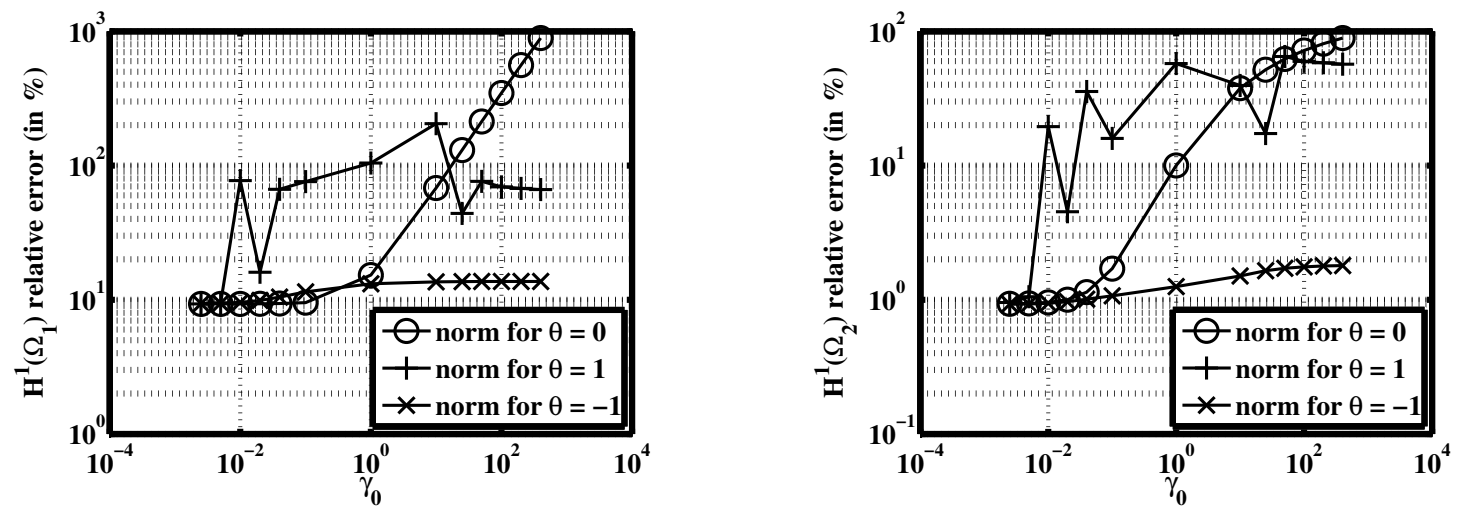

Figure 13: Influence of $\gamma_{0}$ on the relative $H^{1}$-norm of the error on $\Omega_{1}$ (on the left) and on $\Omega_{2}$ (on the right) in $2 \mathrm{D}$ for $h=1 / 90$ and $P_{2}$ elements.

\subsection{Numerical experiments in the $3 \mathrm{D}$ case}

Due to the high number of degrees of freedom in 3D, it obviously has not been possible to produce convergence curves with a mesh size as small as in $2 \mathrm{D}$. The convergence curves for $3 \mathrm{D}$ are shown in Figures 14, 15 and 16 only for $P_{1}$ elements. Although we also made some tests with $P_{2}$ elements and on the influence of $\gamma_{0}$, we do not reproduce them for brevity of the paper. Indeed, the conclusions that can be drawn are were very similar to the $2 \mathrm{D}$ case.

\section{Conclusion}

In this paper, we developed a fictitious domain approach for the approximation in small deformations of the frictionless contact with nonzero initial gap of two elastic bodies. The main ingredients are the adaptation of Nitsche's method for the contact condition introduced in $[5,6]$ and the fictitious domain method (inspired by the X-fem) developed in [16] including the stabilization proposed for the elements having a small intersection with the real domains. 

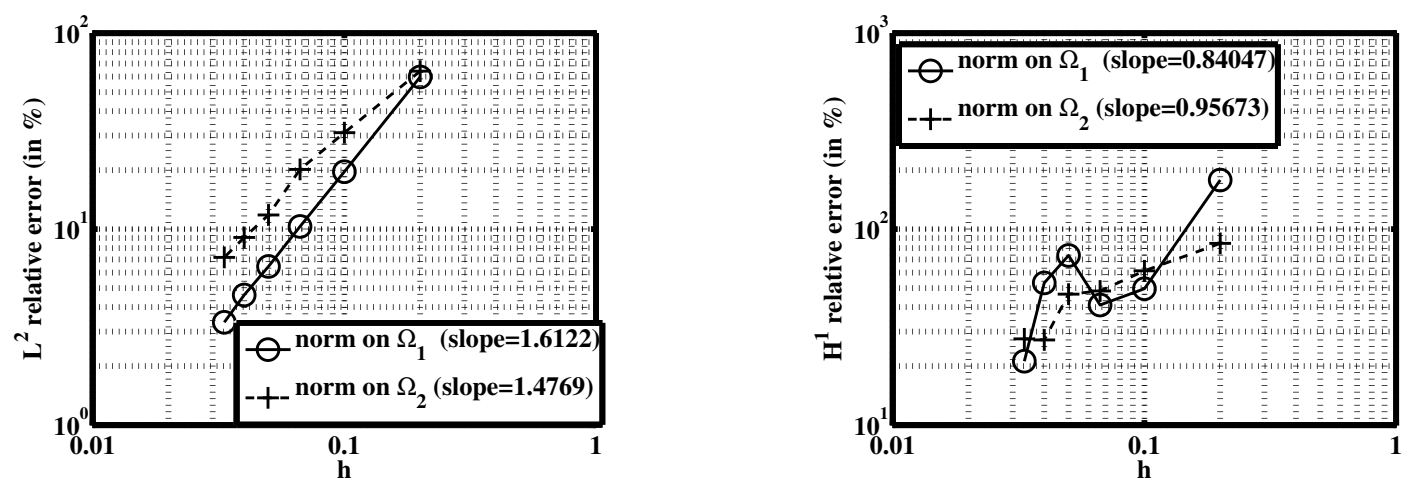

Figure 14: Convergence curves in 3D for the method $\theta=1$, with $\gamma_{0}=1 / 100$ and $P 1$ finite elements for the relative $L^{2}$-norm of the error (on the left) and the relative $H^{1}$-norm of the error (on the right).
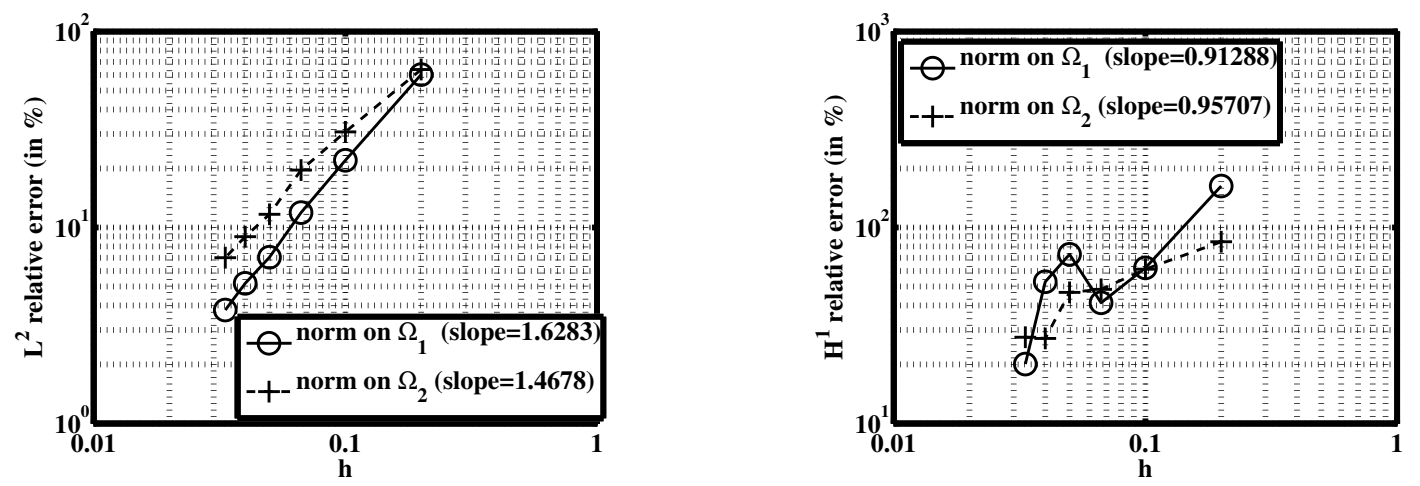

Figure 15: Convergence curves in 3D for the method $\theta=0$, with $\gamma_{0}=1 / 100$ and $P 1$ finite elements for the relative $L^{2}$-norm of the error (on the left) and the relative $H^{1}$-norm of the error (on the right).
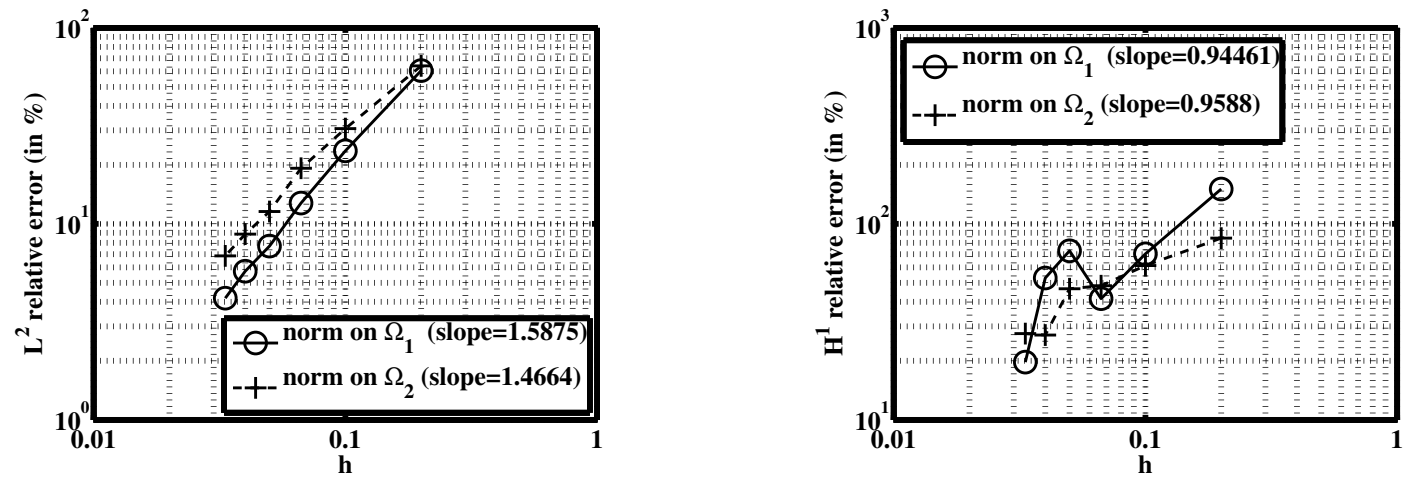

Figure 16: Convergence curves in 3D for the method $\theta=-1$, with $\gamma_{0}=1 / 100$ and $P 1$ finite elements for the relative $L^{2}$-norm of the error (on the left) and the relative $H^{1}$-norm of the error (on the right).

Perspective of this works would be to weakened the conditions on the projection operator $\Pi$ to include for instance non regular situations such as the one illustrated in Figure 17 where $\Pi$ is 
only piecewise regular. Another possibility would be to consider a non-orthogonal projection.

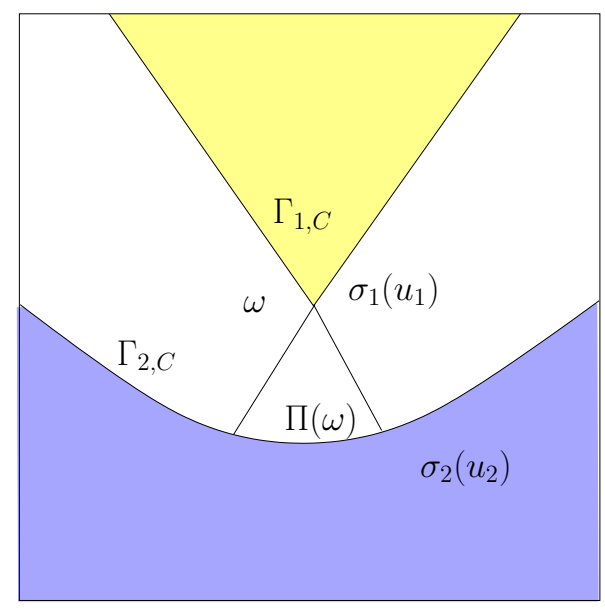

Figure 17: Example of non regular situation on $\Gamma_{1, C}$.

As already mentioned, the analysis can be easily adapted to Tresca friction similarly as it has been done in [7] for the non-fictitious domain situation.

From this study we conclude that the presented method allow an optimal approximation of unilateral contact problems for affine and quadratic finite element methods. The method for $\theta=1$ is symmetric which can be an advantage for the numerical solving but requires a very small parameter $\gamma_{0}$ which may lead to a very stiff discrete problem (10). The method for $\theta=0$ has the advantage of the simplicity and allows the use of a moderate $\gamma_{0}$. Finally, the skew-symmetric method $\theta=-1$ allows the use of larger value of $\gamma_{0}$ which can be a real advantage for the solving of the discrete problem.

\section{Appendix}

Proof of lemma 4.2. First, we define the following matrix norms:

$$
|\|A\||_{\infty, \hat{K}}=\sup _{x \in \hat{K}}\left(|\|A(x)\||_{F}\right) \quad \text { and } \quad|\|A\||_{2, \hat{K}}^{2}=\int_{\hat{K}}|\|A(x)\||_{F}^{2} \mathrm{~d} x
$$

where $|\|\cdot\||_{F}$ is Frobenius' norm. If $v$ is a fixed vector, we define the translation of a vector $\mathrm{u}$, by $t_{v}(u)=u+v$. In the following, the constant $C$ may vary from a line to another but is independent of $h$. In order to prove (13), we distinguish the three different cases from the definition of $R_{\hat{\rho}}$. First, by using the geometric transformation, the integral is expressed on the reference element. Then by using the equivalence of the infinity norm with the 2-norm located on a ball, we are able to deal with the 2-norm located on the current element. Finally by using the definition of the stress tensor, we obtain the result.

- If K satisfies $\exists \hat{y}_{K}>0$ such that $B\left(\hat{y}_{K}, \hat{\rho}\right) \subset T_{K}^{-1}\left(K \cap \Omega_{1}\right)$, then $\left.R_{\hat{\rho}}\left(u^{h}\right)\right|_{K}=\sigma_{n}\left(\left.u_{1}^{h}\right|_{K}\right)$ and it holds:

$$
\left\|R_{\hat{\rho}}\left(u^{h}\right)\right\|_{0, \Gamma_{1, C} \cap K}^{2}=\int_{\Gamma_{1, C} \cap K} \sigma_{n}\left(u_{1}^{h}\right)^{2} \mathrm{~d} \Gamma .
$$


We define $\hat{\Gamma}_{1}=T_{K}^{-1}\left(\Gamma_{1, C} \cap K\right)$ and $\hat{\sigma}\left(u_{1}\right)=\sigma\left(u_{1}^{h}\right) \circ T_{K}$ and $\hat{n}_{1}$ a unit normal vector on $\hat{\Gamma}_{1, C}$.

$$
\begin{aligned}
\int_{\Gamma_{1, C} \cap K} \sigma_{n}\left(u_{1}^{h}\right)^{2} \mathrm{~d} \Gamma & =\int_{\hat{\Gamma}_{1}} \hat{\sigma}_{n}\left(u_{1}\right)^{2}\left|\operatorname{det}\left(J_{K}\right)\right|\left\|J_{K}^{-1} \hat{n}_{1}\right\| \mathrm{d} \hat{\Gamma} \\
& =\int_{\hat{\Gamma}_{1}}\left|\hat{\sigma}\left(u_{1}\right) n \cdot n\right|^{2}\left|\operatorname{det}\left(J_{K}\right)\right|\left\|J_{K}^{-1} \hat{n}_{1}\right\| \mathrm{d} \hat{\Gamma} \\
& \leqslant C h_{K}^{d-1}\left|\left\|\hat{\sigma}\left(u_{1}\right)\right\|\right|_{\infty, \hat{K}}^{2}\left|\hat{\Gamma}_{1}\right| .
\end{aligned}
$$

because

$$
\left|\hat{\sigma}\left(u_{1}\right) n \cdot n\right| \leqslant\left|\left\|\hat{\sigma}\left(u_{1}\right)\right\|\right|_{F}\|n\|_{2}^{2}=\left|\left\|\hat{\sigma}\left(u_{1}\right)\right\|\right|_{F} .
$$

Moreover, $|\hat{\Gamma}|_{1}$ is bounded, indeed the operator $T_{K}$ is a continuous one to one correspondence. Now using the equivalence of norms in $P^{k}(\hat{K})^{d}$, we have:

$$
\begin{aligned}
\left|\left\|\hat{\sigma}\left(u_{1}\right)\right\|\right|_{\infty, \hat{K}}^{2} & \leqslant\left|\left\|\hat{\sigma}\left(u_{1}\right)\right\|\right|_{\infty, B\left(\hat{y}_{K}, 2\right)}^{2}=\left|\left\|\hat{\sigma}\left(u_{1}\right) \circ t_{-\hat{y}_{K}}\right\|\right|_{\infty, B(0,2)}^{2} \\
& \leqslant C\left|\left\|\hat{\sigma}\left(u_{1}\right) \circ t_{-\hat{y}_{K}}\right\|\right|_{2, B(0, \hat{\rho})}^{2}=C\left|\left\|\hat{\sigma}\left(u_{1}\right)\right\|\right|_{2, B\left(\hat{y}_{K}, \hat{\rho}\right)}^{2} \\
& \leqslant C\left|\left\|\hat{\sigma}\left(u_{1}\right)\right\|\right|_{\left.2, T_{K}^{-1}\left(\Omega_{1} \cap K\right)\right)}^{2}=C \int_{T_{K}^{-1}\left(\Omega_{1} \cap K\right)}\left|\left\|\hat{\sigma}\left(u_{1}\right)\right\|\right|_{F}^{2} \mathrm{~d} \hat{x} .
\end{aligned}
$$

Using the upper bound of $\left|\hat{\Gamma}_{1}\right|$ and the previous inequalities, it holds:

$$
\begin{aligned}
\int_{\Gamma_{1, C} \cap K} R_{\hat{\rho}}\left(u^{h}\right)^{2} \mathrm{~d} \Gamma & \leqslant C \frac{h_{K}^{d-1}}{h_{K}^{d}} \int_{T_{K}^{-1}\left(\Omega_{1} \cap K\right)}\left|\left\|\hat{\sigma}\left(u_{1}\right)\right\|\right|_{F}^{2}\left|\operatorname{det}\left(J_{K}\right)\right| \mathrm{d} \hat{x} \\
& \leqslant C h_{K}^{-1} \int_{\Omega_{1} \cap K}\left|\left\|\sigma\left(u_{1}^{h}\right)\right\|\right|_{F}^{2} \mathrm{~d} x \\
& \leqslant\left. C h_{K}^{-1} \int_{\Omega_{1} \cap K}\left\|A \nabla u_{1}^{h}\right\|\right|_{F} ^{2} \mathrm{~d} x \\
& \leqslant C h_{K}^{-1} \int_{\Omega_{1} \cap K}\left\|\nabla u_{1}^{h}\right\|_{2}^{2} \mathrm{~d} x .
\end{aligned}
$$

- Otherwise, if $\exists \widetilde{K} \in S_{K}$ such as $\exists \hat{y}_{\widetilde{K}}>0$ such that $B\left(\hat{y}_{\widetilde{K}}, \hat{\rho}\right) \subset T_{\widetilde{K}}^{-1}\left(\widetilde{K} \cap \Omega_{2}\right)$, then $\left.R_{\hat{\rho}}\left(v^{h}\right)\right|_{K}=\sigma_{n}\left(E_{\widetilde{K}}\left(v_{2}^{h}\right) \circ \Pi\right)|\operatorname{det}(\nabla P i)|$ and using the continuous of $J_{\Pi}$ i.e. $\left|\operatorname{det}\left(J_{\Pi}\right)\right| \leqslant C$, it holds:

$$
\begin{aligned}
\left\|R_{\hat{\rho}}\left(u^{h}\right)\right\|_{0, \Gamma_{1, C} \cap K}^{2} & =\int_{\Gamma_{1, C} \cap K} \sigma_{n}\left(E_{\widetilde{K}}\left(u_{2}^{h}\right) \circ \Pi\right)^{2}\left|\operatorname{det}\left(J_{\Pi}\right)\right|^{2} \mathrm{~d} \Gamma \\
& \leqslant C \int_{\Gamma_{1, C} \cap K} \sigma_{n}\left(E_{\widetilde{K}}\left(u_{2}^{h}\right) \circ \Pi\right)^{2}\left|\operatorname{det}\left(J_{\Pi}\right)\right| \mathrm{d} \Gamma \\
& \leqslant C \int_{\Pi_{\left(\Gamma_{1, C} \cap K\right)}} \sigma_{n}\left(E_{\widetilde{K}}\left(u_{2}^{h}\right)\right)^{2} \mathrm{~d} \Gamma \\
& \leqslant C \int_{\bigcup_{\bar{K} \in S_{K}} \Gamma_{2, C} \cap \bar{K}} \sigma_{n}\left(E_{\widetilde{K}}\left(u_{2}^{h}\right)\right)^{2} \mathrm{~d} \Gamma .
\end{aligned}
$$

We define $\hat{\Gamma}_{2}=T_{\widetilde{K}}^{-1}\left(\bigcup_{\bar{K} \in S_{K}} \Gamma_{2, C} \cap \bar{K}\right)$ and $\hat{\sigma}\left(u_{2}\right)=\sigma\left(u_{2}^{h}\right) \circ T_{\widetilde{K}}$ and $\hat{n}_{2}$ a unit normal vector on $\hat{\Gamma}_{2, C}$. As previously, we have $\left|\hat{\Gamma}_{2}\right|$ bounded. In the same way as in (32), we have:

$$
\int_{\bigcup_{\bar{K} \in S_{K}} \Gamma_{2, C} \cap \bar{K}} \sigma_{n}\left(E_{\widetilde{K}}\left(u_{2}^{h}\right)\right)^{2} \mathrm{~d} \Gamma \leqslant C h_{\widetilde{K}}^{d-1}\left|\left\|\sigma\left(\hat{u}_{2}\right)\right\|\right|_{\infty, \hat{\Gamma}_{2}}^{2}\left|\hat{\Gamma}_{2}\right| .
$$


Now using the equivalence of norms in $P^{k}(\hat{K})^{d}$ and in the same way as in (33), we obtain:

$$
\begin{aligned}
\left|\left\|\sigma\left(\hat{u}_{2}\right)\right\|\right|_{\infty, \hat{\Gamma}_{2}}^{2} & \leqslant\left|\left\|\sigma\left(\hat{u}_{2}\right)\right\|\right|_{\infty, B\left(\hat{y}_{\widetilde{K}}, 2\|\Pi\|\left\|T_{\widetilde{K}}^{-1}\right\|\right)}^{2} \\
& \leqslant C \int_{T_{\widetilde{K}}^{-1}\left(\Omega_{1} \cap \widetilde{K}\right)}\left|\left\|\sigma\left(\hat{u}_{2}\right)\right\|\right|_{F}^{2} \mathrm{~d} \hat{x} .
\end{aligned}
$$

Hence, using the previous inequalities, it holds:

$$
\begin{aligned}
\int_{\Gamma_{1, C} \cap K} R_{\hat{\rho}}\left(u^{h}\right)^{2} \mathrm{~d} \Gamma & \leqslant C \frac{h_{\widetilde{K}}^{d-1}}{h_{\widetilde{K}}^{d}} \int_{T_{\widetilde{K}}^{-1}\left(\Omega_{1} \cap \widetilde{K}\right)}\left|\left\|\hat{\sigma}\left(u_{1}\right)\right\|\right|_{F}^{2}\left|\operatorname{det}\left(J_{\widetilde{K}}\right)\right| \mathrm{d} \hat{x} \\
& \leqslant C h_{\widetilde{K}}^{-1} \int_{\Omega_{1} \cap \widetilde{K}}\left\|\nabla u_{2}^{h}\right\|_{2}^{2} \mathrm{~d} x .
\end{aligned}
$$

- Otherwise, we suppose it exits an neighbor element $K^{\prime}$ of $K$ such that $\exists \hat{y}_{K^{\prime}}>0$ such that $B\left(\hat{y}_{K^{\prime}}, \hat{\rho}\right) \subset T_{K^{\prime}}^{-1}\left(K^{\prime} \cap \Omega_{1}\right)$, then $\left.R_{\hat{\rho}}\left(v^{h}\right)\right|_{K}=\sigma_{n}\left(E_{K^{\prime}}\left(v_{1}^{h}\right)\right)$. Then, it holds:

$$
\left\|R_{\hat{\rho}}\left(u^{h}\right)\right\|_{0, \Gamma_{1, C} \cap K}^{2}=\int_{\Gamma_{1, C} \cap K} \sigma_{n}\left(E_{K^{\prime}}\left(u_{1}^{h}\right)\right)^{2} \mathrm{~d} \Gamma
$$

We define by $\hat{\Gamma}_{1}^{\prime}=T_{K^{\prime}}^{-1}\left(\Gamma_{1, C} \cap K\right)$ and $\hat{\sigma}^{\prime}\left(u_{1}\right)=\sigma\left(u_{1}^{h}\right) \circ T_{K^{\prime}}$ and by $\hat{n}_{1}$ a unit normal vector on $\hat{\Gamma}_{1, C}$. As previously, we have $\left|\hat{\Gamma}_{1}^{\prime}\right|$ bounded. In the same way as in (32), we have:

$$
\begin{aligned}
\int_{\Gamma_{1, C} \cap K} \sigma_{n}\left(E_{K^{\prime}}\left(u_{1}^{h}\right)\right)^{2} \mathrm{~d} \Gamma & =\int_{\hat{\Gamma}_{1}^{\prime}} \hat{\sigma}^{\prime}\left(u_{1}\right)^{2}\left|\operatorname{det}\left(J_{K^{\prime}}\right)\right|\left\|J_{K^{\prime}}^{-1} \hat{n}_{1}\right\| \mathrm{d} \hat{\Gamma} \\
& \leqslant C h_{K^{\prime}}^{d-1}\left|\left\|\hat{\sigma}^{\prime}\left(u_{1}\right)\right\|\right|_{\infty, T_{K^{\prime}}^{-1}(K)}^{2}\left|\hat{\Gamma}_{1}^{\prime}\right| .
\end{aligned}
$$

Now using the equivalence of norms in $P^{k}(\hat{K})^{d}$ and in the same way as in (33), we have:

$$
\begin{aligned}
\left.\left\|\sigma\left(\hat{u}_{1}^{\prime}\right)\right\|\right|_{\infty, T_{K^{\prime}}(K)} ^{2} & \leqslant\left|\left\|\sigma\left(\hat{u}_{1}^{\prime}\right)\right\|\right|_{\infty, B\left(\hat{y}_{K^{\prime}}, 4\right)}^{2} \\
& \leqslant C \int_{T_{K^{\prime}}^{-1}\left(\Omega_{1} \cap K^{\prime}\right)}\left\|\sigma\left(\hat{u}_{1}^{\prime}\right)\right\|_{F}^{2} \mathrm{~d} \hat{x} .
\end{aligned}
$$

Hence, using the previous inequalities, it holds:

$$
\begin{aligned}
\int_{\Gamma_{1, C} \cap K} R_{\hat{\rho}}\left(u^{h}\right)^{2} \mathrm{~d} \Gamma & \leqslant C \frac{h_{K^{\prime}}^{d-1}}{h_{K^{\prime}}^{d}} \int_{T_{K^{\prime}}^{-1}\left(\Omega_{1} \cap K^{\prime}\right)}\left|\left\|\sigma\left(\hat{u}_{1}^{\prime}\right)\right\|\right|_{F}^{2}\left|\operatorname{det}\left(J_{K^{\prime}}\right)\right| \mathrm{d} \hat{x} \\
& \leqslant C h_{K^{\prime}}^{-1} \int_{\Omega_{1} \cap K^{\prime}}\left\|\nabla u_{1}^{h}\right\|_{2}^{2} \mathrm{~d} x .
\end{aligned}
$$

Finally, by iterating on all the elements $K$ intersecting $\Gamma_{1, C}$ and using the quasi uniformity of the mesh, we obtain (13).

Proof of lemma 4.7. We argue by contradiction. It is sufficient to prove the result for $\gamma=M$. Suppose there exists $\left(v_{n}\right)_{n \in \mathbb{N}} \subset V$ such that $\sum_{i=1,2}\left\|v_{i, n}\right\|_{1, \Omega_{i}}^{2}=1$, for $n \in \mathbb{N}$, which satisfies

$$
a\left(v_{n}, v_{n}\right)+\frac{1}{2} \sum_{i=1,2} \int_{\Gamma_{i, D}} M^{-1} v_{i, n}^{2} \mathrm{~d} \Gamma \leqslant \frac{1}{n} .
$$


Hence, it holds $\lim _{n \rightarrow+\infty} \sum_{i=1,2} \int_{\Gamma_{i, D}} v_{i, n}^{2} \mathrm{~d} \Gamma=0$ and $\lim _{n \rightarrow+\infty} a\left(v_{n}, v_{n}\right)=0$. From the weak sequential compactness of the unit ball of $V$, there exits $v \in V$ and a subsequence still denoted by $v_{n}$ which weakly converges to $v$. The compact injection of $H^{1}$ into $L^{2}$ implies that up to a subsequence, $v_{n}$ converges to $v$ strongly in $L^{2}\left(\Omega_{1}\right)^{d} \times L^{2}\left(\Omega_{2}\right)^{d}$. First, we show that $v=0$ and then that $v_{n}$ converges to $v$ strongly in $H^{1}\left(\Omega_{1}\right)^{d} \times H^{1}\left(\Omega_{2}\right)^{d}$. By using the lower semi-continuity of $v \mapsto$ $\sum_{i=1,2} \int_{\Gamma_{i, D}} v_{i, n}^{2} \mathrm{~d} \Gamma$, we have $\sum_{i=1,2} \int_{\Gamma_{i, D}} v_{i}^{2} \mathrm{~d} \Gamma=0$ with mes $\left(\Gamma_{i, D}\right) \neq 0$. Furthermore, due to the $L^{2}$-convergence, one has $\lim _{n \rightarrow+\infty} \sum_{i=1,2}\left\|v_{i, n}\right\|_{0, \Omega_{i}}=\sum_{i=1,2}\left\|v_{i}\right\|_{0, \Omega_{i}}$. Similarly by using the weak lower semi-continuity of $a(.,$.$) , we deduce a(v, v)=0$ and using the property of the fourth order tensor $A$, it holds:

$$
0=\int_{\Omega_{i}} \sigma\left(v_{i}\right): \varepsilon\left(v_{i}\right) \mathrm{d} \Omega=\int_{\Omega_{i}} \varepsilon\left(v_{i}\right): \varepsilon\left(v_{i}\right) \mathrm{d} \Omega=\left\|\varepsilon\left(v_{i}\right)\right\|_{0, \Omega_{i}} .
$$

Let us finally show that $v=0$. Since, the tensor $A$ is uniformly elliptic, it holds:

$$
a\left(v_{n}, v_{n}\right) \geqslant C \sum_{i=1,2} \int_{\Omega_{i}} \varepsilon\left(v_{i, n}\right): \varepsilon\left(v_{i, n}\right) \mathrm{d} \Omega=C \sum_{i=1,2}\left\|\varepsilon\left(v_{i, n}\right)\right\|_{0, \Omega_{i}} \quad \text { and } \lim _{n \rightarrow+\infty} a\left(v_{n}, v_{n}\right)=0 .
$$

Hence

$$
\lim _{n \rightarrow+\infty} \sum_{i=1,2}\left\|\varepsilon\left(v_{i, n}\right)\right\|_{0, \Omega_{i}}=\sum_{i=1,2}\left\|\varepsilon\left(v_{i}\right)\right\|_{1, \Omega_{i}}=0 .
$$

Moreover, thanks to Korn's inequality (see [9]), it holds:

$$
\sum_{i=1,2}\left\|\varepsilon\left(v_{i, n}\right)\right\|_{0, \Omega_{i}}+\sum_{i=1,2}\left\|v_{i, n}\right\|_{0, \Gamma_{i, D}} \geqslant C \sum_{i=1,2}\left\|v_{i, n}\right\|_{0, \Omega_{i}} .
$$

We deduce:

$$
\lim _{n \rightarrow+\infty} \sum_{i=1,2}\left\|v_{i, n}\right\|_{1, \Omega_{i}}=\sum_{i=1,2}\left\|v_{i}\right\|_{1, \Omega_{i}}=0
$$

which contradicts $\sum_{i=1,2}\left\|v_{i, n}\right\|_{1, \Omega_{i}}^{2}=1$.

\section{Proof for operator $B^{h}$ to be hemi-continuous (for the proof of Theorem 4.1).}

First, we need to prove $B^{h}$ is coercive which is a consequence of the previous lemmas. Then we establish an estimate which will imply the hemi-continuity. Let $u^{h}, v^{h} \in V^{h}$, it holds:

$$
\left(B^{h} u^{h}-B^{h} v^{h}, u^{h}-v^{h}\right)_{1, \Omega}=I+I I+I I I
$$

with $I=a\left(u^{h}-v^{h}, u^{h}-v^{h}\right)-\int_{\Gamma_{1, C}} \theta \gamma R_{\hat{\rho}}\left(u^{h}-v^{h}\right) R_{\hat{\rho}}\left(u^{h}-v^{h}\right) \mathrm{d} \Gamma$

$$
\begin{gathered}
-\sum_{i=1,2} \int_{\Gamma_{i, D}} \theta \gamma \bar{R}_{\hat{\rho}}\left(u_{i}^{h}-v_{i}^{h}\right) \cdot \bar{R}_{\hat{\rho}}\left(u_{i}^{h}-v_{i}^{h}\right) \mathrm{d} \Gamma, \\
I I=\sum_{i=1,2} \int_{\Gamma_{i, D}} \frac{1}{\gamma}\left(\bar{P}_{i, \gamma}^{h, \hat{\rho}}\left(u_{i}^{h}\right)-\bar{P}_{i, \gamma}^{h, \hat{\rho}}\left(v_{i}^{h}\right)\right) \cdot\left(\bar{P}_{i, \gamma \theta}^{h, \hat{\rho}}\left(u_{i}^{h}\right)-\bar{P}_{i, \gamma \theta}^{h, \hat{\rho}}\left(v_{i}^{h}\right)\right) \mathrm{d} \Gamma, \\
I I I=\int_{\Gamma_{1, C}} \frac{1}{\gamma}\left(\left[P_{\gamma}^{h, \hat{\rho}}\left(u^{h}\right)-g\right]_{+}-\left[P_{\gamma}^{h, \hat{\rho}}\left(v^{h}\right)-g\right]_{+}\right)\left(P_{\theta \gamma}^{h, \hat{\rho}}\left(u^{h}\right)-P_{\theta \gamma}^{h, \hat{\rho}}\left(v^{h}\right)\right) \mathrm{d} \Gamma .
\end{gathered}
$$

Now, we need to bound $I, I I, I I I$ from below to prove the coercivity. 
Using Young's inequality for $\beta>0$, it holds:

$$
\begin{aligned}
I I I \geqslant & \left(1-\frac{|1-\theta|}{2 \beta}\right)\left\|\gamma^{-\frac{1}{2}}\left(\left[P_{\gamma}^{h, \hat{\rho}}\left(u^{h}\right)-g\right]_{+}-\left[P_{\gamma}^{h, \hat{\rho}}\left(v^{h}\right)-g\right]_{+}\right)\right\|_{0, \Gamma_{1, C}}^{2} \\
& -\frac{|1-\theta| \beta}{2}\left\|\gamma^{\frac{1}{2}} R_{\hat{\rho}}\left(u^{h}-v^{h}\right)\right\|_{0, \Gamma_{1, C}}^{2}
\end{aligned}
$$

Using Young's inequality for $\beta^{\prime}>0$ :

$$
\begin{aligned}
I I \geqslant & \left(1-\frac{|1+\theta|}{2 \beta^{\prime}}\right) \sum_{i=1,2}\left\|\gamma^{-\frac{1}{2}}\left(u_{i}^{h}-v_{i}^{h}\right)\right\|_{\Gamma_{i, D}}^{2} \\
& +\left(\theta-\frac{|1+\theta| \beta^{\prime}}{2}\right) \sum_{i=1,2}\left\|\gamma^{\frac{1}{2}}\left(\bar{R}_{\hat{\rho}}\left(u_{i}^{h}\right)-\bar{R}_{\hat{\rho}}\left(v_{i}^{h}\right)\right)\right\|_{\Gamma_{i, D}}^{2} .
\end{aligned}
$$

We deduced from the estimates of $I I$ and $I I I$ that:

$$
\begin{aligned}
& \left(B^{h} u^{h}-B^{h} v^{h}, u^{h}-v^{h}\right)_{1, \Omega} \\
\geqslant & a\left(u^{h}-v^{h}, u^{h}-v^{h}\right)-\theta\left\|\gamma^{\frac{1}{2}} R_{\hat{\rho}}\left(u^{h}-v^{h}\right)\right\|_{0, \Gamma_{1, C}}^{2}-\theta \sum_{i=1,2}\left\|\gamma^{\frac{1}{2}}\left(\bar{R}_{\hat{\rho}}\left(u_{i}^{h}\right)-\bar{R}_{\hat{\rho}}\left(v_{i}^{h}\right)\right)\right\|_{\Gamma_{i, D}}^{2} \\
& +\left(1-\frac{|1-\theta|}{2 \beta}\right)\left\|\gamma^{-\frac{1}{2}}\left(\left[P_{\gamma}^{h, \hat{\rho}}\left(u^{h, \hat{\rho}}\right)-g\right]_{+}-\left[P_{\gamma}^{h, \hat{\rho}}\left(v^{h}\right)-g\right]_{+}\right)\right\|_{0, \Gamma_{1, C}}^{2} \\
& -\frac{|1-\theta| \beta}{2}\left\|\gamma^{\frac{1}{2}} R_{\hat{\rho}}\left(u^{h}-v^{h}\right)\right\|_{0, \Gamma_{1, C}}^{2} \\
& +\left(1-\frac{|1+\theta|}{2 \beta^{\prime}}\right) \sum_{i=1,2}\left\|\gamma^{-\frac{1}{2}}\left(u_{i}^{h}-v_{i}^{h}\right)\right\|_{\Gamma_{i, D}}^{2}+\left(\theta-\frac{|1+\theta| \beta^{\prime}}{2}\right) \sum_{i=1,2}\left\|\gamma^{\frac{1}{2}}\left(\bar{R}_{\hat{\rho}}\left(u_{i}^{h}\right)-\bar{R}_{\hat{\rho}}\left(v_{i}^{h}\right)\right)\right\|_{\Gamma_{i, D}}^{2} \\
\geqslant & a\left(u^{h}-v^{h}, u^{h}-v^{h}\right)+\left(1-\frac{|1+\theta|}{2 \beta^{\prime}}\right) \sum_{i=1,2}\left\|\gamma^{-\frac{1}{2}}\left(u_{i}^{h}-v_{i}^{h}\right)\right\|_{\Gamma_{i, D}}^{2} \\
& -\frac{|1+\theta| \beta^{\prime}}{2} \sum_{i=1,2}\left\|\gamma^{\frac{1}{2}}\left(\bar{R}_{\hat{\rho}}\left(u_{i}^{h}\right)-\bar{R}_{\hat{\rho}}\left(v_{i}^{h}\right)\right)\right\|_{\Gamma_{i, D}}^{2}-\left(\theta+\frac{|1-\theta| \beta}{2}\right)\left\|\gamma^{\frac{1}{2}} R_{\hat{\rho}}\left(u^{h}-v^{h}\right)\right\|_{0, \Gamma_{1, C}}^{2} \\
& +\left(1-\frac{|1-\theta|}{2 \beta}\right)\left\|\gamma^{-\frac{1}{2}}\left(\left[P_{\gamma}^{h, \hat{\rho}}\left(u^{h}\right)-g\right]_{+}-\left[P_{\gamma}^{h, \hat{\rho}}\left(v^{h}\right)-g\right]_{+}\right)\right\|_{0, \Gamma_{1, C}}^{2} \cdot
\end{aligned}
$$

If $\theta=1$ and $\beta^{\prime}=2$, we have:

$$
\begin{aligned}
& \left(B^{h} u^{h}-B^{h} v^{h}, u^{h}-v^{h}\right)_{1, \Omega} \\
\geqslant & a\left(u^{h}-v^{h}, u^{h}-v^{h}\right)+\frac{1}{2} \sum_{i=1,2}\left\|\gamma^{-\frac{1}{2}}\left(u_{i}^{h}-v_{i}^{h}\right)\right\|_{\Gamma_{i, D}}^{2}-2 \sum_{i=1,2}\left\|\gamma^{\frac{1}{2}}\left(\bar{R}_{\hat{\rho}}\left(u_{i}^{h}\right)-\bar{R}_{\hat{\rho}}\left(v_{i}^{h}\right)\right)\right\|_{\Gamma_{i, D}}^{2} \\
& +\left\|\gamma^{-\frac{1}{2}}\left(\left[P_{\gamma}^{h, \hat{\rho}}\left(u^{h}\right)-g\right]_{+}-\left[P_{\gamma}^{h, \hat{\rho}}\left(v^{h}\right)-g\right]_{+}\right)\right\|_{0, \Gamma_{1, C}}^{2}-\left\|\gamma^{\frac{1}{2}} R_{\hat{\rho}}\left(u^{h}-v^{h}\right)\right\|_{0, \Gamma_{1, C}}^{2} .
\end{aligned}
$$

Thus, if $\gamma_{0}$ is sufficiently small and using the coercivity (14) for $I$ and the previous lemma 4.7:

$$
\left(B^{h} u^{h}-B^{h} v^{h}, u^{h}-v^{h}\right)_{1, \Omega} \geqslant C \sum_{i=1,2}\left\|u_{i}^{h}-v_{i}^{h}\right\|_{1, \Omega_{i}}^{2} .
$$

If $\theta=-1$, choose $\beta=\frac{|1-\theta|}{2}$, it holds:

$$
\begin{aligned}
& \left(B^{h} u^{h}-B^{h} v^{h}, u^{h}-v^{h}\right)_{1, \Omega} \\
\geqslant & a\left(u^{h}-v^{h}, u^{h}-v^{h}\right)+\sum_{i=1,2}\left\|\gamma^{-\frac{1}{2}}\left(u_{i}^{h}-v_{i}^{h}\right)\right\|_{\Gamma_{i, D}}^{2}
\end{aligned}
$$


and from the coercivity (14) for $I$, we obtain:

$$
\left(B^{h} u^{h}-B^{h} v^{h}, u^{h}-v^{h}\right)_{1, \Omega} \geqslant C \sum_{i=1,2}\left\|u_{i}^{h}-v_{i}^{h}\right\|_{1, \Omega_{i}}^{2} .
$$

If $\theta \neq-1$, we take $\beta=\frac{|1-\theta|}{2}$ and $\beta^{\prime}=|1+\theta|$, it holds:

$$
\begin{aligned}
& \left(B^{h} u^{h}-B^{h} v^{h}, u^{h}-v^{h}\right)_{1, \Omega} \\
\geqslant & a\left(u^{h}-v^{h}, u^{h}-v^{h}\right)+\frac{1}{2} \sum_{i=1,2}\left\|\gamma^{-\frac{1}{2}}\left(u_{i}^{h}-v_{i}^{h}\right)\right\|_{\Gamma_{i, D}}^{2}-\frac{(1+\theta)^{2}}{2} \sum_{i=1,2}\left\|\gamma^{\frac{1}{2}}\left(\bar{R}_{\hat{\rho}}\left(u_{i}^{h}\right)-\bar{R}_{\hat{\rho}}\left(v_{i}^{h}\right)\right)\right\|_{\Gamma_{i, D}}^{2} \\
& -\frac{1}{4}(1+\theta)^{2}\left\|\gamma^{\frac{1}{2}} R_{\hat{\rho}}\left(u^{h}-v^{h}\right)\right\|_{0, \Gamma_{1, C}}^{2} .
\end{aligned}
$$

So, using $\gamma_{0}$ sufficiently small and using the coercivity (14) for $I$ and previous lemma 4.7, it holds:

$$
\left(B^{h} u^{h}-B^{h} v^{h}, u^{h}-v^{h}\right)_{1, \Omega} \geqslant C \sum_{i=1,2}\left\|u_{i}^{h}-v_{i}^{h}\right\|_{1, \Omega_{i}}^{2} .
$$

Now, we prove the hemi-continuity of $B^{h}$. Let $t, s \in[0,1]$ and $u^{h}, v^{h} \in V^{h}$, we have:

$$
\begin{aligned}
& \left|\left(B^{h}\left(u^{h}-t v^{h}\right)-B^{h}\left(u^{h}-s v^{h}\right), v^{h}\right)_{1, \Omega}\right| \\
\leqslant & |s-t| a\left(v^{h}, v^{h}\right)+\left.|s-t||\theta||| \gamma^{\frac{1}{2}} R_{\hat{\rho}}\left(v^{h}\right)\right|_{\Gamma_{1, C}} ^{2}+|s-t||\theta| \sum_{i=1,2}\left\|\gamma^{\frac{1}{2}} \bar{R}_{\hat{\rho}}\left(v_{i}^{h}\right)\right\|_{\Gamma_{i, D}}^{2} \\
& +|s-t| \sum_{i=1,2} \int_{\Gamma_{i, D}} \frac{1}{\gamma}\left|\bar{P}_{i, \gamma}^{h, \hat{\rho}}\left(v_{i}^{h}\right)\right| \cdot\left|\bar{P}_{i, \gamma \theta}^{h, \hat{\rho}}\left(v_{i}^{h}\right)\right| \mathrm{d} \Gamma \\
& +\int_{\Gamma_{1, C}} \frac{1}{\gamma}\left|\left[P_{\gamma}^{h, \hat{\rho}}\left(u^{h}-t v^{h}\right)-g\right]_{+}-\left[P_{\gamma}^{h, \hat{\rho}}\left(u^{h}-s v^{h}\right)-g\right]_{+}\right|\left|P_{\theta \gamma}^{h, \hat{\rho}}\left(v^{h}\right)\right| \mathrm{d} \Gamma .
\end{aligned}
$$

For all $a, b$ in $\mathbb{R}$, we have the following estimate:

$$
\left|[a]_{+}-[b]_{+}\right| \leqslant|a-b| .
$$

So we deduce that

$$
\begin{aligned}
& \int_{\Gamma_{1, C}} \frac{1}{\gamma}\left|\left[P_{\gamma}^{h, \hat{\rho}}\left(u^{h}-t v^{h}\right)-g\right]_{+}-\left[P_{\gamma}^{h, \hat{\rho}}\left(u^{h}-s v^{h}\right)-g\right]_{+}\right|\left|P_{\theta \gamma}^{h, \hat{\rho}}\left(v^{h}\right)\right| \mathrm{d} \Gamma \\
\leqslant & |s-t| \int_{\Gamma_{1, C}} \frac{1}{\gamma}\left|\llbracket v^{h} \cdot n \rrbracket-\gamma R_{\hat{\rho}}\left(v^{h}\right)\right|\left|P_{\theta \gamma}^{h, \hat{\rho}}\left(v^{h}\right)\right| \mathrm{d} \Gamma .
\end{aligned}
$$

Hence

$$
\begin{aligned}
& \left|\left(B^{h}\left(u^{h}-t v^{h}\right)-B^{h}\left(u^{h}-s v^{h}\right), v^{h}\right)_{1, \Omega}\right| \\
\leqslant & |s-t|\left(a\left(v^{h}, v^{h}\right)+|\theta||| \gamma^{\frac{1}{2}} R_{\hat{\rho}}\left(v^{h}\right)\left\|_{\Gamma_{1, C}}^{2}+|\theta| \sum_{i=1,2}\right\| \gamma^{\frac{1}{2}} \bar{R}_{\hat{\rho}}\left(v_{i}^{h}\right) \|_{\Gamma_{i, D}}^{2}\right. \\
& \left.+\sum_{i=1,2} \int_{\Gamma_{i, D}} \frac{1}{\gamma}\left|\bar{P}_{i, \gamma}^{h, \hat{\rho}}\left(v_{i}^{h}\right)\right| \cdot\left|\bar{P}_{i, \gamma \theta}^{h, \hat{\rho}}\left(v_{i}^{h}\right)\right| \mathrm{d} \Gamma+\int_{\Gamma_{1, C}} \frac{1}{\gamma}\left|P_{\gamma}^{h, \hat{\rho}}\left(v^{h}\right)\right|\left|P_{\theta \gamma}^{h, \hat{\rho}}\left(v^{h}\right)\right| \mathrm{d} \Gamma\right) .
\end{aligned}
$$

Hence $B^{h}$ is hemi-continuous. 


\section{References}

[1] C. Annavarapu, M. Hautefeuille and J. E. Dolbow. A robust Nitsche's formulation for interface problems. Comput. Methods Appl. Mech. Engrg., 225-228(2012), pp. 44-54.

[2] S. Bertoluzza, M. Ismail and B. Maury. The fat boundary method: Semi-discrete scheme and some numerical experiments. In: Domain Decomposition Methods in Science and Engineering, vol. 40 of Lecture Notes in Computational Science and Engineering, Springer Berlin Heidelberg, 2005, pp. 513-520.

[3] Haïm Brézis. quations et inquations non linaires dans les espaces vectoriels en dualit. Ann. Inst. Fourier, 18:1 (1968), pp. 115-175.

[4] E. Burman and P. Hansbo. Fictitious domain finite element methods using cut elements: II. A stabilized Nitsche's method. Comput. Methods Appl. Mech. Engrg., 62:4(2012), pp. 328-341.

[5] F. Chouly and P. Hild. A Nitsche-based method for unilateral contact problems: numerical analysis. SIAM J. Numer. Anal., 51:2(2013), pp. 1295-1307.

[6] F. Chouly, P. Hild and Y. Renard. Symmetric and non-symmetric variants of Nitsche's method for contact problems in elasticity: theory and numerical experiments. To appear in Math. Comp.

[7] F. Chouly. An adaptation of Nitsche's method to the Tresca friction problem. J. Math. Anal. Appl., 411(2014), pp. 329-339.

[8] P. G. Ciarlet. The Finite Element Method for Elliptic Problems. North-Holland, 1978.

[9] G. Duvaut and J. L. Lions. Les inquations en mcanique et en physique. Dunod, Paris, 1972.

[10] G. Fichera. Problemi elastostatici con vincoli unilaterali: Il problema di Signorini con ambigue condizioni al contorno. Atti Accad. Naz. Lincei Mem. Cl. Sci. Fis. Mat. Natur., I:8(1963), pp. 91-140.

[11] A. Fritz, S. Hüeber and B.I. Wohlmuth. A comparison of mortar and Nitsche techniques for linear elasticity. Calcolo, 41:3(2004), pp. 115-137.

[12] R. Glowinski and Y. Kuznetsov. On the solution of the Dirichlet problem for linear elliptic operators by a distributed Lagrange multiplier method. C. R. Acad. Sci. Paris Sér. I Math., 327:7 (1998), pp. 693-698.

[13] V. Girault and R. Glowinski. Error analysis of a fictitious domain method applied to a Dirichlet problem. Japan J. Indust. Appl. Math., 12:3(1995), pp. 487-514.

[14] A. Hansbo and P. Hansbo. An unfitted finite element method, based on Nitsche's method, for elliptic interface problems. Comput. Methods Appl. Mech. Engrg., 191(2002), pp. 5537-5552.

[15] J. Haslinger, I. Hlaváček, and J. Nečas. Numerical methods for unilateral problems in solid mechanics. Handbook of Numerical Analysis (eds. P.G. Ciarlet and J.L. Lions). Vol. IV (1996), North Holland, pp. 313-385.

[16] J. Haslinger and Y. Renard. A new fictitious domain approach inspired by the extended finite element method. SIAM J. Numer. Anal., 47(2009), pp. 1474-1499. 
[17] M. Juntunen and R. Stenberg. Nitsche's method for general boundary conditions. Math. Comp., 78:267(2009), pp. 1353-1374.

[18] N. Kikuchi and J. T. Oden. Contact problems in elasticity: a study of variational in- equalities and finite element methods. Studies in Applied Mathematics, vol. 8, SIAM, Philadelphia, 1988.

[19] G. I. Marchuk. Methods of numerical mathematics, vol. 2 of Applications of Mathematics, 2nd ed., Springer-Verlag, New York, 1982.

[20] N. Möes, E. Béchet and M. Tourbier. Imposing Dirichlet boundary conditions in the extended finite element method. Internat. J. Numer. Methods Engrg., 67:12(2006), pp. 1641-1669.

[21] N. Möes, J. Dolbow and T. Belytschko. A finite element method for cracked growth without remeshing. Internat. J. Numer. Methods Engrg., 46(1999), pp. 131-150.

[22] M. Moussaoui and K. Khodja. Regularité des solutions d un problème mêlé DirichletSignorini dans un domaine polygonal plan. Commun. Partial Differential Equations, 17 (1992), pp. $805-826$.

[23] S. Nicaise, Y. Renard and E. Chahine. Optimal convergence analysis for the eXtended Finite Element, Internat. J. Numer. Methods Engrg., 86(2011), pp. 528-548.

[24] J. Nitsche. Über ein Variationsprinzip zur Lsung von Dirichlet-Problemen bei Verwendung von Teilrumen, die keinen Randbedingungen unterworfen sind. Abhandlungen aus dem Mathematischen Seminar der Universitt Hamburg, 36:1(1971), pp. 9-15.

[25] C. S. Peskin. The immersed boundary method. Acta Numer., 11 (2002), pp. 479-517.

[26] E. Pierres, M.C. Baietto and A. Gravouil. A two-scale extended finite element method for modeling 3D crack growth with interfacial contact. Comput. Methods Appl. Mech. Eng., 199(2010), pp. 1165-1177.

[27] Y. Renard. Generalized Newton's methods for the approximation and resolution of frictional contact problems in elasticity. Comput. Methods Appl. Mech. Eng., 256(2013), pp. 38-55.

[28] B. Wohlmuth. Variationally consistent discretization schemes and numerical algorithms for contact problems. Acta Numerica, 20(2011), pp. 569-734. 